\title{
EM- and JMAP-ML Based Joint Estimation Algorithms for Robust Wireless Geolocation in Mixed LOS/NLOS Environments
}

\author{
Feng Yin, Carsten Fritsche, Fredrik Gustafsson and Abdelhak M. Zoubir
}

\section{Linköping University Post Print}

\section{Tweet}

N.B.: When citing this work, cite the original article.

(C2014 IEEE. Personal use of this material is permitted. However, permission to reprint/republish this material for advertising or promotional purposes or for creating new collective works for resale or redistribution to servers or lists, or to reuse any copyrighted component of this work in other works must be obtained from the IEEE.

Feng Yin, Carsten Fritsche, Fredrik Gustafsson and Abdelhak M. Zoubir, EM- and JMAP-ML Based Joint Estimation Algorithms for Robust Wireless Geolocation in Mixed LOS/NLOS Environments, 2014, IEEE Transactions on Signal Processing, (62), 1, 168-182.

http://dx.doi.org/10.1109/TSP.2013.2286779

Postprint available at: Linköping University Electronic Press http://urn.kb.se/resolve?urn=urn:nbn:se:liu:diva-104838 


\title{
EM- and JMAP-ML Based Joint Estimation Algorithms for Robust Wireless Geolocation in Mixed LOS/NLOS Environments
}

\author{
Feng Yin, Student Member, IEEE, Carsten Fritsche, Member, IEEE, Fredrik Gustafsson, Fellow, IEEE \\ and Abdelhak M. Zoubir, Fellow, IEEE
}

\begin{abstract}
We consider robust geolocation in mixed line-ofsight (LOS)/non-LOS (NLOS) environments in cellular radio networks. Instead of assuming known propagation channel states (LOS or NLOS), we model the measurement error with a general two-mode mixture distribution although it deviates from the underlying error statistics. To avoid offline calibration, we propose to jointly estimate the geographical coordinates and the mixture model parameters. Two iterative algorithms are developed based on the well-known expectation-maximization (EM) criterion and joint maximum a posteriori-maximum likelihood (JMAP-ML) criterion to approximate the ideal maximumlikelihood estimator (MLE) of the unknown parameters with low computational complexity. Along with concrete examples, we elaborate the convergence analysis and the complexity analysis of the proposed algorithms. Moreover, we numerically compute the Cramér-Rao lower bound (CRLB) for our joint estimation problem and present the best achievable localization accuracy in terms of the CRLB. Various simulations have been conducted based on a real-world experimental setup, and the results have shown that the ideal MLE can be well approximated by the JMAP-ML algorithm. The EM estimator is inferior to the JMAPML estimator but outperforms other competitors by far.
\end{abstract}

Index Terms-Cramér-Rao lower bound (CRLB), EM criterion, geolocation, JMAP-ML criterion, mixture distribution, nonline-of-sight (NLOS) mitigation.

\section{INTRODUCTION}

The problem of position estimation has been well studied in line-of-sight (LOS) environments, i.e., in the case of Gaussian measurement error. In harsh situations, such as indoor environments and some urban areas, however, the measurement error is non-Gaussian and heavy-tailed, which may seriously degrade the estimation performance of conventional techniques (see e.g., [1]-[7]) developed under the Gaussian error assumption.

In this paper, we take a mixture distribution to characterize the non-Gaussian and heavy-tailed nature of the measurement

Copyright (C)2013 IEEE. Personal use of this material is permitted. However, permission to use this material for any other purposes must be obtained from the IEEE by sending a request to pubs-permissions@iee.org.

F. Yin and A. M. Zoubir are with the Signal Processing Group, Institute of Telecommunications, Technische Universität Darmstadt, Darmstadt, 64283, Germany (e-mail: fyin@spg.tu-darmstadt.de; zoubir@spg.tu-darmstadt.de).

C. Fritsche was with the Department of Electrical Engineering, Division of Automatic Control, Linköping University, Linköping, SE-581 83, Sweden. He is now with the IFEN GmbH, Poing, 85586, Germany (e-mail: carsten@isy.liu.se).

F. Gustafsson is with the Department of Electrical Engineering, Division of Automatic Control, Linköping University, Linköping, SE-581 83, Sweden (e-mail: fredrik@isy.liu.se). error. This has been experimentally validated in different scenarios. For instance, round-trip time-of-arrival (RTOA) measurements were collected in different indoor environments around the MIT campus, and the measurement errors was reported to well match a Gaussian mixture distribution with three components under both LOS and non-LOS (NLOS) conditions [8]. In [9], it was corroborated that measurement errors well fit a contaminated Gaussian model for both an indoor situation (anechoic chamber) and an outdoor situation (parking lot). In [10], field-trial results demonstrated that RTOA measurement errors suit a two-mode Gaussian mixture distribution in an operating cellular radio network in a German city center.

Designing a robust position estimator under the nonGaussian and heavy-tailed measurement error assumption is not an easy task. Amongst all kinds of position estimators toward that end, the maximum-likelihood estimator (MLE) is most attractive due to its notable asymptotic performance [11]. However, we may confront two challenges in obtaining an MLE in practice. First, the probability density function (PDF) of the measurement error distribution is unknown, and so is the log-likelihood function, from which the MLE shall be resolved. Secondly, the regular ML estimation is cumbersome, implying tremendous efforts for solving the MLE. Many efforts have been paid to find alternatives, which are easy to implement and meanwhile capable of approximating or reproducing the MLE with rather low computational complexity. In the sequel, we show two examples in the context of robust geolocation.

The first challenge can be overcome by performing a nonparametric PDF estimation step prior to the regular ML estimation. This idea was initially proposed for robust multiuser detection in impulsive noise channels in [12] and revisited for robust TOA based geolocation in mixed LOS/NLOS environments in [13]. Therein, the developed robust iterative nonparametric (RIN) algorithm seeks to approximate the actual measurement error PDF (albeit unknown) using the adaptive kernel density estimation (AKDE) and update the position estimate in an attempt to maximize the approximated log-likelihood function. These two steps iterate until a given convergence condition is met. Simulation results demonstrated that the RIN position estimator approaches the MLE as the number of measurements increases, whereas it deviates from the MLE with small measurement sample size due to the poor performance of the AKDE in this case. The computational 
complexity of the RIN algorithm is moderate and meets the demand of E-911 services by far.

In this paper, we reconsider the geolocation problem in [13] except that we explicitly appoint a specific two-mode mixture distribution to represent the measurement error. To avoid offline calibration, we set the mixture model parameters to be unknown. Our task is to jointly estimate the position and the mixture model parameters. This joint parameter estimation problem, considered afterwards, falls in the second challenge. As we will see later, the log-likelihood function contains "the log of the sum", implying difficulties in obtaining the MLE through standard approaches. Instead, we apply the expectation-maximization (EM) criterion [14] and the joint maximum a posteriori- maximum likelihood (JMAP-ML) criterion [15] to approximate the MLE. The EM criterion is well known due to its ability to reproduce the MLE. Hence, there exist many works (see e.g., [16]-[19]) developed based on it. However, the JMAP-ML criterion is less popular, mainly because the produced estimator is biased and inconsistent [15]. The optimality of the developed EM algorithm will be impaired when the underlying measurement error statistics deviates from the assumed model, for instance, in our geolocation problem. As we will see later, the JMAPML algorithm would outperform the EM algorithm in that case.

For clarity, we summarize our original contributions of this paper as follows:

- We apply EM- and JMAP-ML criteria, respectively, to a specific NLOS mitigation problem and consequently develop two iterative algorithms for approximating the ideal MLE of the unknown parameters with low computational complexity;

- Along with concrete examples, we carry out convergence analysis and complexity analysis of the proposed algorithms.

- We numerically compute the Cramér-Rao lower bound (CRLB) for our joint estimation problem.

The remaining parts of this paper are organized as follows. In Section II, we introduce the signal model and state the problem at hand. In Section III, we show the difficulties involved in implementing the ideal MLE in practical applications and give our solution. In Section IV, we introduce two iterative algorithms to approximate the ideal MLE. Section $\mathrm{V}$ further elaborates on the algorithm implementation, the convergence analysis, and the complexity analysis with concrete examples. In Section VI, we perform CRLB analysis and show the best achievable localization accuracy, followed by a comprehensive simulation based on a real-world experimental setup in Section VII. Finally, Section VIII concludes the paper.

Notation: Throughout this paper, matrices are presented with boldface uppercase letters and vectors with boldface lowercase letters. $\mathbb{Z}$ denotes the set of integers. The operator $[\cdot]^{T}$ stands for vector/matrix transpose, while $\mathbf{X} \succeq \mathbf{Y}$ means that the matrix difference $\mathbf{X}-\mathbf{Y}$ is positive semidefinite. The symbol $\mathbf{1}$ denotes a column vector of all ones. The operator $\mathbb{E}_{p(x)}(\cdot)$ denotes the statistical expectation taken with respect to the PDF $p(x)$. The operator $\ln (\cdot)$ denotes the natural logarithm. Further, $\nabla_{\boldsymbol{\theta}}=\partial / \partial \boldsymbol{\theta}$ denotes the gradient operator and $\Delta_{\boldsymbol{\theta}}^{\boldsymbol{\theta}}=\nabla_{\boldsymbol{\theta}} \nabla_{\boldsymbol{\theta}}^{T}$ denotes the Laplace operator. Lastly, the operator $\operatorname{dim}(\mathbf{a})$ denotes the dimension of vector $\mathbf{a}$.

\section{Signal MODEL}

We consider a scenario where $N$ base stations (BSs) surround a stationary mobile station (MS) of interest in a cellular network. Let $\boldsymbol{x}_{i}=\left[x_{i}, y_{i}\right]^{T} i=1,2, \ldots, N$ be the a priori known geographical coordinates of the $i$ th BS and let $\boldsymbol{x}=[x, y]^{T}$ be the unknown geographical coordinates of the MS. ${ }^{1}$ For each BS, we obtain $K(K \geq 1)$ range measurement(s) and subsequently relay them to a fusion center for post-processing [20].

The BSs and the MS are assumed to be precisely synchronized in time. As a consequence, the $k$ th range measurement collected at the $i$ th $\mathrm{BS}, r_{i, k}$, is expressed by

$$
r_{i, k}=\underbrace{\sqrt{\left(x-x_{i}\right)^{2}+\left(y-y_{i}\right)^{2}}}_{d_{i}(\boldsymbol{x})}+v_{i, k}
$$

where $d_{i}(\boldsymbol{x})$ represents the exact Euclidean distance between the MS and the $i$ th $\mathrm{BS}$; and $v_{i, k}$ denotes the measurement error associated with the $i$ th $\mathrm{BS}$ and the $k$ th time instance.

In this paper, we focus on geolocation of a single user in mixed LOS/NLOS scenarios, where the channel state for each BS-MS pair can be either in LOS or in NLOS. Moreover, the channel state stays unaltered during the measurement collection (quasi-stationary for at least $K$ samples). The measurement error distribution is represented by $p_{V}^{(1)}\left(v ; \boldsymbol{\beta}_{1}\right)$ under LOS condition whereas $p_{V}^{(2)}\left(v ; \boldsymbol{\beta}_{2}\right)$ under NLOS condition. Throughout the paper, we make the following assumptions: (1) the sources of error stem from propagation channel, not from the hardware [21]; (2) the two measurement error distributions belong to designated families; (3) environmental parameters $\boldsymbol{\beta}_{1}$ and $\boldsymbol{\beta}_{2}$ are unknown so as to get rid of the offline calibration usually performed prior to the geolocation. The reasons for the last assumption are twofold. On the one hand, offline calibration is expensive. On the other hand, the underlying environmental parameters responsible for the measurement collection mostly likely differ from those measured "long time ago" in the offline calibration.

For better readability, we express our signal model in a compact vector form as follows:

$$
\mathbf{r}=\mathbf{h}(\boldsymbol{x})+\mathbf{v}
$$

where

$$
\begin{aligned}
\mathbf{r} & =\left[r_{1,1}, \ldots, r_{1, K}, \ldots, r_{N, 1}, \ldots, r_{N, K}\right]^{T}, \\
\mathbf{h}(\boldsymbol{x}) & =[\underbrace{d_{1}(\boldsymbol{x}), \ldots, d_{1}(\boldsymbol{x})}_{K \text { repetitions }}, \ldots, \underbrace{d_{N}(\boldsymbol{x}), \ldots, d_{N}(\boldsymbol{x})}_{K \text { repetitions }}]^{T}, \\
\mathbf{v} & =\left[v_{1,1}, \ldots, v_{1, K}, \ldots, v_{N, 1}, \ldots, v_{N, K}\right]^{T} .
\end{aligned}
$$

Column vectors $\mathbf{r}, \mathbf{h}(\boldsymbol{x})$ and $\mathbf{v}$ are all of dimension $N K \times 1$.

\footnotetext{
${ }^{1}$ Our algorithm can be straightforwardly extended to three-dimensional case.
} 


\section{Ideal Maximum-Likelihood Estimation: Challenges AND SOLUTION}

In this paper, we pursue the maximum-likelihood estimator for its famous asymptotic property [11, Theorem 7.3]. The maximum-likelihood estimator of the unknown parameters, $\boldsymbol{\theta}=\left[\boldsymbol{\beta}_{1}^{T}, \boldsymbol{\beta}_{2}^{T}, \boldsymbol{x}^{T}\right]^{T}$, given the precise knowledge about the channel states, can be solved through globally maximizing the log-likelihood function

$$
\begin{aligned}
\ln (\tilde{p}(\mathbf{r} ; \boldsymbol{\theta})) & =\ln \left(\prod_{i=1}^{M} \prod_{k=1}^{K} p_{V}^{(1)}\left(r_{i, k}-d_{i}(\boldsymbol{x}) ; \boldsymbol{\beta}_{1}\right)\right. \\
\cdot & \left.\prod_{i=M+1}^{N} \prod_{k=1}^{K} p_{V}^{(2)}\left(r_{i, k}-d_{i}(\boldsymbol{x}) ; \boldsymbol{\beta}_{2}\right)\right) \\
= & \sum_{i=1}^{M} \sum_{k=1}^{K} \ln p_{V}^{(1)}\left(r_{i, k}-d_{i}(\boldsymbol{x}) ; \boldsymbol{\beta}_{1}\right) \\
& +\sum_{i=M+1}^{N} \sum_{k=1}^{K} \ln p_{V}^{(2)}\left(r_{i, k}-d_{i}(\boldsymbol{x}) ; \boldsymbol{\beta}_{2}\right)
\end{aligned}
$$

with respect to $\boldsymbol{\theta}$. In (6), we assume, without loss of generality, the first $M$ BSs are LOS BSs while the rest $N-M$ BSs are NLOS BSs. ${ }^{2}$ Unfortunately the knowledge of the channel states is rarely available without running any channel identification procedure, which hinders the practical use of this ideal estimator. NLOS identification can be done, for instance, by investigating some features extracted from the received waveform or by directly comparing the received waveform with known patterns [22]. The former requires the knowledge of $\boldsymbol{\beta}_{1}$ and $\boldsymbol{\beta}_{2}$, while the latter is workable only in some certain situations. Alternatively, we could carry out the ML estimation in (6) for every hypothesis characterized by the subset of BSs that are assumed to be in NLOS with the MS (and the subset of the BSs in LOS with the MS) and ultimately choose the estimate with the highest likelihood. This idea is similar to the one proposed in [23]. Apart from the possibility of missing the right hypothesis, the overwhelming drawback of this method is the high computational complexity.

To avoid the above drawbacks, we seek for alternative algorithms with the following features:

- no offline calibration of the environmental parameters,

- simultaneous determination of the positions, the channel states, and the environmental parameters,

- rather low computational complexity.

Our solution is due to the fact that when we treat the available data as a whole in the fusion center, the corresponding measurement error samples can be deemed as coming from two clusters-one for LOS measurements with $M K$ (albeit unknown) samples and the other for NLOS measurements with $(N-M) K$ samples. To simplify our estimation problem, the measurement error is represented by a two-mode mixture distribution

$$
p_{V}(v)=\sum_{l=1}^{2} \alpha_{l} p_{V}^{(l)}\left(v ; \boldsymbol{\beta}_{l}\right)
$$

\footnotetext{
${ }^{2}$ Here, (N)LOS BS is the short notation of a BS in (N)LOS with the MS.
}

where $\alpha_{1}=M K / N K=M / N$ and $\alpha_{2}=(N-M) K / N K=$ $(N-M) / N$ are unknown. Despite the sub-optimality of our assumed model, we can then apply both the EM criterion and the JMAP-ML criterion to reach our goal.

\section{Alternative Maximum-Likehood Estimation: GENERAL RESUlTS}

Based on the signal model in (1) and the newly assumed noise model in (7), a new $\log$-likelihood function of $\boldsymbol{\theta}=$ $[\underbrace{\alpha_{1}, \alpha_{2}, \boldsymbol{\beta}_{1}^{T}, \boldsymbol{\beta}_{2}^{T}}_{\boldsymbol{\theta}_{m}^{T}}, \boldsymbol{x}^{T}]^{T}$ is given by

$$
\begin{aligned}
\mathcal{L}_{I}(\boldsymbol{\theta} ; \mathbf{r}) & =\ln (p(\mathbf{r} ; \boldsymbol{\theta}))=\ln \left(\prod_{i=1}^{N} \prod_{k=1}^{K} p\left(r_{i, k} ; \boldsymbol{\theta}\right)\right) \\
& =\sum_{i=1}^{N} \sum_{k=1}^{K} \ln \left(\sum_{l=1}^{2} \alpha_{l} p_{V}^{(l)}\left(r_{i, k}-d_{i}(\boldsymbol{x}) ; \boldsymbol{\beta}_{l}\right)\right)
\end{aligned}
$$

and the MLE $\hat{\boldsymbol{\theta}}_{\mathrm{ML}}$ is obtained through solving

$$
\begin{array}{cl}
\underset{\boldsymbol{\theta}}{\arg \max } & \mathcal{L}_{I}(\boldsymbol{\theta} ; \mathbf{r}) \\
\text { subject to } & \alpha_{1} \geq 0, \alpha_{2} \geq 0, \\
& \alpha_{1}+\alpha_{2}=1, \\
& \text { and constraints on } \boldsymbol{\beta}_{l}, \quad l=1,2 .
\end{array}
$$

It is noteworthy to mention that the unknown parameters $\boldsymbol{\theta}$ should not be confused with the one defined for the ideal case.

The cost function in (9) is cumbersome, thus we take a detour to approximate the MLE with hopefully low computational complexity. To that end, we introduce a complete data set $\mathbf{z}=\{\mathbf{y}, \mathbf{r}\}$ with $\mathbf{y}=$ $\left[y_{1,1}, \cdots, y_{1, K}, \cdots, y_{N, 1}, \cdots, y_{N, K}\right]^{T}$ being a vector of $N K$ random variables (also called latent variables) whose value tell us which mixture component has generated the corresponding measurement error. More specifically, one can define $y_{i, k} \in\{1,2\}$ and set $y_{i, k}=1$ if $v_{i, k}$ is generated by the first mixture component $p_{V}^{(1)}\left(v ; \boldsymbol{\beta}_{1}\right)$ or $y_{i, k}=2$ otherwise. The complete data log-likelihood function is easily expressed by

$$
\begin{aligned}
\mathcal{L}_{C}(\boldsymbol{\theta} ; \mathbf{y}, \mathbf{r}) & =\ln (p(\mathbf{y}, \mathbf{r} ; \boldsymbol{\theta}))=\ln \left(\prod_{i=1}^{N} \prod_{k=1}^{K} p\left(y_{i, k}, r_{i, k} ; \boldsymbol{\theta}\right)\right) \\
& =\sum_{i=1}^{N} \sum_{k=1}^{K} \ln \left(\alpha_{y_{i, k}} p_{V}^{\left(y_{i, k}\right)}\left(r_{i, k}-d_{i}(\boldsymbol{x}) ; \boldsymbol{\beta}_{y_{i, k}}\right)\right)
\end{aligned}
$$

where the second equality relies on the assumption that $r_{i, k}$ 's are independent and as a consequence $y_{i, k}$ 's are also independent. To avoid ambiguity, we refer to the original loglikelihood function $\mathcal{L}_{I}(\boldsymbol{\theta} ; \mathbf{r})$ in (8) as incomplete data loglikelihood function.

It is clear that the newly introduced complete data loglikelihood function $\mathcal{L}_{C}(\boldsymbol{\theta} ; \mathbf{y}, \mathbf{r})$ has a more tractable form, from which the MLE shall be approximated either via the EM criterion or via the JMAP-ML criterion. In what follows, the resulting algorithms are called "EM algorithm" and "JMAPML algorithm", respectively. 


\section{A. EM Algorithm}

As it is well known, the idea behind the EM criterion is to estimate the unknown parameters iteratively in two steps-an expectation (E)-step and a maximization (M)-step. In the first step, i.e., the E-step, statistical expectation of the complete data log-likelihood is taken with respect to the conditional probability of the latent variables. In the second step, i.e., the M-step, the conditional expectation obtained above is maximized with respect to the parameters of interest. The two steps iterate until a predetermined convergence condition is met. Given the a priori parameter estimate $\boldsymbol{\theta}^{(\eta)}$, we show in the sequel the work-flow of the proposed EM algorithm on the $(\eta+1)$ th iteration.

The first step (E-step): Let us first define the conditional expectation of the complete data log-likelihood as follows:

$$
Q\left(\boldsymbol{\theta} ; \boldsymbol{\theta}^{(\eta)}\right)=\sum_{\mathbf{y}} \ln (p(\mathbf{y}, \mathbf{r} ; \boldsymbol{\theta})) \operatorname{Pr}\left\{\mathbf{y} \mid \mathbf{r} ; \boldsymbol{\theta}^{(\eta)}\right\} .
$$

Following similar steps as given in [24] for simplifying $Q\left(\boldsymbol{\theta} ; \boldsymbol{\theta}^{(\eta)}\right)$, we obtain

$Q\left(\boldsymbol{\theta} ; \boldsymbol{\theta}^{(\eta)}\right)=\sum_{i=1}^{N} \sum_{k=1}^{K} \sum_{l=1}^{2} \ln \left(\alpha_{l} p_{V}^{(l)}\left(r_{i, k}-d_{i}(\boldsymbol{x}) ; \boldsymbol{\beta}_{l}\right)\right) \tilde{P}_{i, k, l}^{(\eta)}$

where $\tilde{P}_{i, k, l}^{(\eta)}$ is a short-hand notation of the conditional probability $\operatorname{Pr}\left\{y_{i, k}=l \mid r_{i, k} ; \boldsymbol{\theta}^{(\eta)}\right\}$, which can be computed by means of Bayes' rule as follows:

$$
\begin{aligned}
\tilde{P}_{i, k, l}^{(\eta)} & =\operatorname{Pr}\left\{y_{i, k}=l \mid r_{i, k} ; \boldsymbol{\theta}^{(\eta)}\right\} \\
& =\frac{\alpha_{l}^{(\eta)} p_{V}^{(l)}\left(r_{i, k}-d_{i}\left(\boldsymbol{x}^{(\eta)}\right) ; \boldsymbol{\beta}_{l}^{(\eta)}\right)}{p\left(r_{i, k} ; \boldsymbol{\theta}^{(\eta)}\right)}
\end{aligned}
$$

with

$$
p\left(r_{i, k} ; \boldsymbol{\theta}^{(\eta)}\right)=\sum_{l=1}^{2} \alpha_{l}^{(\eta)} p_{V}^{(l)}\left(r_{i, k}-d_{i}\left(\boldsymbol{x}^{(\eta)}\right) ; \boldsymbol{\beta}_{l}^{(\eta)}\right) .
$$

The second step (M-Step): We maximize $Q\left(\boldsymbol{\theta} ; \boldsymbol{\theta}^{(\eta)}\right)$, derived in (12), with respect to the vector parameter $\boldsymbol{\theta}$, and thus obtain on the $(\eta+1)$ th iteration

$$
\boldsymbol{\theta}^{(\eta+1)}=\underset{\boldsymbol{\theta}}{\arg \max } Q\left(\boldsymbol{\theta} ; \boldsymbol{\theta}^{(\eta)}\right)
$$

\section{B. JMAP-ML Algorithm}

As another means of approximating the MLE, we adopt the JMAP-ML criterion, where the complete data log-likelihood function is maximized directly with respect to both $\boldsymbol{\theta}$ and $\mathbf{y}$, that is,

$$
\underset{\boldsymbol{\theta}, \mathbf{y}}{\arg \max } \mathcal{L}_{C}(\boldsymbol{\theta} ; \mathbf{y}, \mathbf{r}) \equiv \underset{\boldsymbol{\theta}}{\arg \max }\left\{\underset{\mathbf{y}}{\arg \max } \mathcal{L}_{C}(\boldsymbol{\theta} ; \mathbf{y}, \mathbf{r})\right\} .
$$

The JMAP-ML criterion, as its name suggests, incorporates an MAP estimation step (in terms of the latent variables in y) and an ML estimation step (in terms of the deterministic parameters in $\boldsymbol{\theta}$ ) [15]. In general, an algorithm developed based on the JMAP-ML criterion starts with a carefully selected initial guess $\boldsymbol{\theta}^{(0)}$ and alternates between the above mentioned two steps in an iterative process. Details about these two steps are shown for our problem in the sequel.

The first step (MAP estimation of y): Let us first re-write the log-likelihood function of the complete data as follows:

$\mathcal{L}_{C}(\boldsymbol{\theta} ; \mathbf{y}, \mathbf{r})=\ln (p(\mathbf{y}, \mathbf{r} ; \boldsymbol{\theta}))=\ln (\operatorname{Pr}\{\mathbf{y} \mid \mathbf{r} ; \boldsymbol{\theta}\})+\ln (p(\mathbf{r} ; \boldsymbol{\theta}))$

where the term $\ln (p(\mathbf{r} ; \boldsymbol{\theta}))$ is independent of $\mathbf{y}$. Replacing $\boldsymbol{\theta}$ with $\boldsymbol{\theta}^{(\eta)}$ in (17) and solving for the MAP estimate of $\mathbf{y}$, yields

$$
\mathbf{y}^{(\eta+1)}=\underset{\mathbf{y}}{\arg \max } \ln \left(\operatorname{Pr}\left\{\mathbf{y} \mid \mathbf{r} ; \boldsymbol{\theta}^{(\eta)}\right\}\right),
$$

which can be slashed into $N K$ simpler pieces as follows:

$$
y_{i, k}^{(\eta+1)}=\underset{y_{i, k}}{\arg \max } \ln \left(\operatorname{Pr}\left\{y_{i, k} \mid r_{i, k} ; \boldsymbol{\theta}^{(\eta)}\right\}\right),
$$

$\forall(i, k) \in \mathcal{S} \triangleq\{(1,1), \cdots,(1, K), \cdots,(N, 1), \cdots,(N, K)\}$ with $\operatorname{Pr}\left\{y_{i, k} \mid r_{i, k} ; \boldsymbol{\theta}^{(\eta)}\right\}$ being calculated in light of (13). Since $y_{i, k}$ is discrete-valued, the global optimal solution to (19) must be the one among $\left\{y_{i, k}=1,2\right\}$ that maximizes $\ln \left(\operatorname{Pr}\left\{y_{i, k} \mid r_{i, k} ; \boldsymbol{\theta}^{(\eta)}\right\}\right)$. Since $\ln (\cdot)$ is a monotonic operation, we need only to compare

$$
\Phi_{i, k, l}^{(\eta)} \triangleq \alpha_{l}^{(\eta)} p_{V}^{(l)}\left(r_{i, k}-d_{i}\left(\boldsymbol{x}^{(\eta)}\right) ; \boldsymbol{\beta}_{l}^{(\eta)}\right), l=1,2 .
$$

We set $y_{i, k}^{(\eta+1)}=1(\mathrm{LOS})$ if $\Phi_{i, k, 1}^{(\eta)} \geq \Phi_{i, k, 2}^{(\eta)}$, or $y_{i, k}^{(\eta+1)}=2$ (NLOS) otherwise.

The second step (ML estimation of $\theta$ ): Substituting the obtained MAP estimate $\mathbf{y}^{(\eta+1)}$ into the complete data loglikelihood $\mathcal{L}_{C}(\boldsymbol{\theta} ; \mathbf{y}, \mathbf{r})$, yields

$$
\begin{aligned}
& \mathcal{L}_{C}\left(\boldsymbol{\theta} ; \mathbf{y}^{(\eta+1)}, \mathbf{r}\right) \\
& =\sum_{i=1}^{N} \sum_{k=1}^{K} \ln \left(\alpha_{y_{i, k}^{(\eta+1)}} p_{V}^{\left(y_{i, k}^{(\eta+1)}\right)}\left(r_{i, k}-d_{i}(\boldsymbol{x}) ; \boldsymbol{\beta}_{y_{i, k}^{(\eta+1)}}\right)\right) \\
& =\sum_{i=1}^{N} \sum_{k=1}^{K} \sum_{l=1}^{2} \ln \left(\alpha_{l} p_{V}^{(l)}\left(r_{i, k}-d_{i}(\boldsymbol{x}) ; \boldsymbol{\beta}_{l}\right)\right) \delta\left(l-y_{i, k}^{(\eta+1)}\right)
\end{aligned}
$$

where

$$
\delta\left(l-y_{i, k}^{(\eta+1)}\right)= \begin{cases}1, & \text { if } l=y_{i, k}^{(\eta+1)} \\ 0, & \text { otherwise }\end{cases}
$$

is Kronecker's delta. In the subsequent step, we maximize $\mathcal{L}_{C}\left(\boldsymbol{\theta} ; \mathbf{y}^{(\eta+1)}, \mathbf{r}\right)$ with respect to $\boldsymbol{\theta}$, and thus obtain on the $(\eta+1)$ th iteration

$$
\boldsymbol{\theta}^{(\eta+1)}=\underset{\boldsymbol{\theta}}{\arg \max } \mathcal{L}_{C}\left(\boldsymbol{\theta} ; \mathbf{y}^{(\eta+1)}, \mathbf{r}\right) .
$$

Remark 1: In fact, one can merge the cost functions (12) and (21) into a general form as follows:

$$
\Lambda^{(\eta)}(\boldsymbol{\theta})=\sum_{i=1}^{N} \sum_{k=1}^{K} \sum_{l=1}^{2} \ln \left(\alpha_{l} p_{V}^{(l)}\left(r_{i, k}-d_{i}(\boldsymbol{x}) ; \boldsymbol{\beta}_{l}\right)\right) w_{i, k, l}^{(\eta)}
$$

Therein, the weighting factor $w_{i, k, l}^{(\eta)}$ is distinguished by

$$
w_{i, k, l}^{(\eta)}=\left\{\begin{array}{cl}
\tilde{P}_{i, k, l}^{(\eta)}, & \text { EM algorithm }, \\
\delta\left(l-y_{i, k}^{(\eta+1)}\right), & \text { JMAP-ML algorithm. }
\end{array}\right.
$$


It is interesting to see that $w_{i, k, l}^{(\eta)}$ corresponds to a "soft fusion" of information in the EM algorithm whereas a "hard fusion" of information in the JMAP-ML algorithm.

Remark 2: Although $w_{i, k, l}^{(\eta)}$ differs in the two algorithms, it is a priori determined (in the first step) and contains no optimization variable, meaning that we could follow the same strategy to optimize (12) and (21). Consequently, the corresponding results differ only by the weighting factors.

Remark 3: When the measurement errors are indeed independently and identically distributed (i.i.d.) according to (7), the EM algorithm is able to reproduce the MLE that globally maximizes the incomplete-data log-likelihood function in (8) [14]. However, the JMAP-ML algorithm merely produces biased and inconsistent estimator [15]. We give one example in Section VII-C to justify the above statement.

Remark 4: For our geolocation problem, the EM algorithm becomes suboptimal in terms of localization accuracy. However, the JMAP-ML algorithm is less influenced. This is because by inserting accurately estimated $y_{i, k}^{(\eta+1)}, \alpha_{l}^{(\eta+1)}$, and $\boldsymbol{\beta}_{l}^{(\eta+1)}$ in (21), the reduced cost function (in terms of the position $\boldsymbol{x}$ only) can well approximate the ideal one with known $\boldsymbol{\beta}_{l}, l=1,2$ in (6).

\section{Alternative Maximum-LiKelihood Estimation: CAse Study}

In this section, we use concrete examples to elaborate our proposed algorithms in terms of:

- implementation of the maximization step (cf. Section V-A),

- convergence property (cf. Section V-B),

- computational complexity (cf. Section V-C).

\section{A. Algorithm Implementation}

Example-I: The first example starts with a GaussianGaussian mixture whose PDF is given by

$$
p_{V}(v)=\alpha_{1} \mathcal{N}(v ; \underbrace{\mu_{1}, \sigma_{1}^{2}}_{\boldsymbol{\beta}_{1}})+\alpha_{2} \mathcal{N}(v ; \underbrace{\mu_{2}, \sigma_{2}^{2}}_{\boldsymbol{\beta}_{2}}) .
$$

Here, we assume $\sigma_{1}^{2}$ and $\sigma_{2}^{2}$ to be strictly larger than zero. The vector parameter to be estimated now becomes $\boldsymbol{\theta}=$ $\left[\alpha_{1}, \alpha_{2}, \mu_{1}, \sigma_{1}^{2}, \mu_{2}, \sigma_{2}^{2}, x, y\right]^{T}$, and the cost function in (24) becomes

$$
\Lambda^{(\eta)}(\boldsymbol{\theta})=\sum_{i=1}^{N} \sum_{k=1}^{K} \sum_{l=1}^{2} \ln \left(\alpha_{l} \mathcal{N}\left(r_{i, k}-d_{i}(\boldsymbol{x}) ; \boldsymbol{\beta}_{l}\right)\right) w_{i, k, l}^{(\eta)}
$$

Before proceeding with the maximization, we re-formulate the cost function $\Lambda^{(\eta)}(\boldsymbol{\theta})$ as

$$
\Lambda^{(\eta)}(\boldsymbol{\theta})=\Lambda_{0}^{(\eta)}\left(\alpha_{1}, \alpha_{2}\right)+\sum_{l=1}^{2} \Lambda_{l}^{(\eta)}\left(\mu_{l}, \sigma_{l}^{2}, \boldsymbol{x}\right)
$$

where

$$
\Lambda_{0}^{(\eta)}\left(\alpha_{1}, \alpha_{2}\right) \triangleq \sum_{i=1}^{N} \sum_{k=1}^{K} \ln \left(\alpha_{1}\right) w_{i, k, 1}^{(\eta)}+\ln \left(\alpha_{2}\right) w_{i, k, 2}^{(\eta)},
$$

and for $l=1,2$,

$$
\begin{aligned}
& \Lambda_{l}^{(\eta)}\left(\mu_{l}, \sigma_{l}^{2}, \boldsymbol{x}\right) \\
& \quad \triangleq \sum_{i=1}^{N} \sum_{k=1}^{K} \ln \left(\mathcal{N}\left(r_{i, k}-d_{i}(\boldsymbol{x}) ; \mu_{l}, \sigma_{l}^{2}\right)\right) w_{i, k, l}^{(\eta)} .
\end{aligned}
$$

First, we maximize $\Lambda^{(\eta)}(\boldsymbol{\theta})$ with respect to the mixture model parameters by following the route shown in [24]. More precisely, we solve the following equations:

$$
\begin{gathered}
\frac{\partial}{\partial \alpha_{l}}\left[\Lambda_{0}^{(\eta)}\left(\alpha_{1}, \alpha_{2}\right)+\lambda\left(\sum_{l=1}^{2} \alpha_{l}-1\right)\right]=0 \\
\frac{\partial}{\partial \mu_{l}}\left[\Lambda_{l}^{(\eta)}\left(\mu_{l}, \sigma_{l}^{2}, \boldsymbol{x}^{(\eta)}\right)\right]=0 \\
\frac{\partial}{\partial \sigma_{l}^{2}}\left[\Lambda_{l}^{(\eta)}\left(\mu_{l}^{(\eta+1)}, \sigma_{l}^{2}, \boldsymbol{x}^{(\eta)}\right)\right]=0 .
\end{gathered}
$$

The solutions are in closed form and they are shown in Table I. Note that $\lambda$ in (31) is the Lagrange multiplier and the influence of $\sigma_{l}^{2}$ in (32) will be canceled out in the calculation.

Next, we update the MS position by

$$
\boldsymbol{x}^{(\eta+1)}=\underset{\boldsymbol{x}}{\arg \max } \sum_{l=1}^{2} \Lambda_{l}^{(\eta)}\left(\mu_{l}^{(\eta+1)}, \sigma_{l}^{2,(\eta+1)}, \boldsymbol{x}\right)
$$

where $\mu_{l}^{(\eta+1)}, \sigma_{l}^{2,(\eta+1)}, l=1,2$ are respectively substitutions of the unknown parameters. After some simple manipulations, the above maximization problem boils down to

$$
\boldsymbol{x}^{(\eta+1)}=\underset{\boldsymbol{x}}{\arg \min } f_{1}(\boldsymbol{x})
$$

where the cost function $f_{1}(\boldsymbol{x})$ is given explicitly in Table I. Unfortunately, a closed form solution does not exist because the term $d_{i}(\boldsymbol{x})$ in $f_{1}(\boldsymbol{x})$ is nonlinear in terms of both $x$ and $y$. Hence, we resort to numerical methods. In order to keep this position update step computationally fast and meanwhile maintain a good numerical result, we adopt here the BFGS quasi-Newton method [25] as it guarantees downhill progress towards the local minimum in each Newton step [26].

Example-II: In the second example, we consider a Gaussian-exponential mixture distribution with its PDF given by

$$
p_{V}(v)=\alpha_{1} \mathcal{N}(v ; \underbrace{\mu_{1}, \sigma_{1}^{2}}_{\boldsymbol{\beta}_{1}})+\alpha_{2} \mathcal{E}(v ; \underbrace{\gamma_{\mathcal{E}}}_{\boldsymbol{\beta}_{2}})
$$

where

$$
\mathcal{E}\left(v ; \gamma_{\mathcal{E}}\right)= \begin{cases}\frac{1}{\gamma_{\mathcal{E}}} \exp \left(\frac{-v}{\gamma_{\mathcal{E}}}\right), & v \geq 0 \\ 0, & v<0\end{cases}
$$

and the distribution parameter $\gamma_{\mathcal{E}}$ is strictly larger than zero. We stick to the same procedure shown above to update the parameter estimate. In this case, $\Lambda_{0}^{(\eta)}\left(\alpha_{1}, \alpha_{2}\right)$ and $\Lambda_{1}^{(\eta)}\left(\mu_{1}, \sigma_{1}^{2}, \boldsymbol{x}\right)$ remain unaltered. However, we need to redefine

$$
\Lambda_{2}^{(\eta)}\left(\gamma_{\mathcal{E}}, \boldsymbol{x}\right) \triangleq \sum_{i=1}^{N} \sum_{k=1}^{K} \ln \left(\mathcal{E}\left(r_{i, k}-d_{i}(\boldsymbol{x}) ; \gamma_{\mathcal{E}}\right)\right) w_{i, k, 2}^{(\eta)}
$$


TABLE I

SUMMARY OF THE PARAMETER UPDATE FOR DIFFERENT CASES

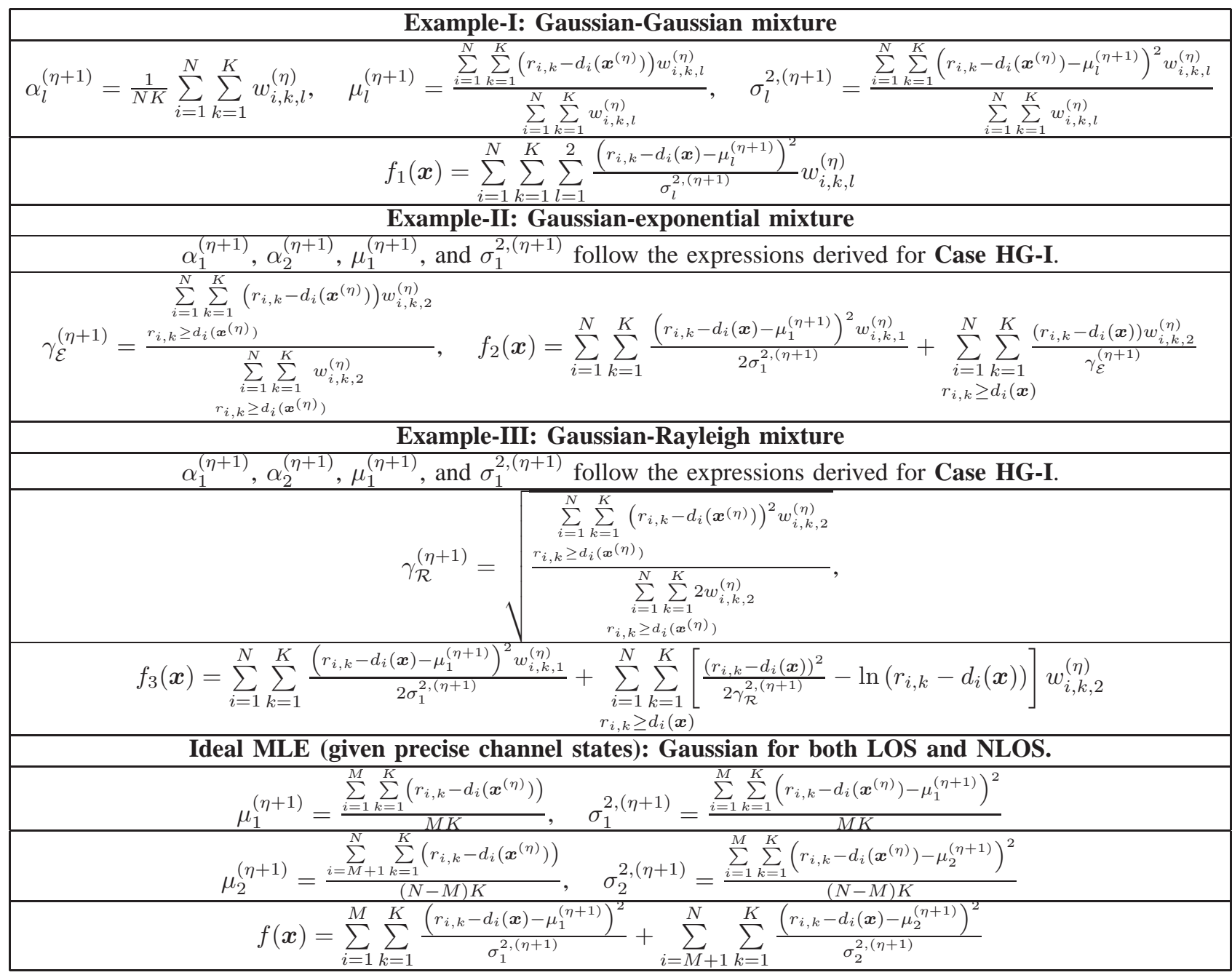

Similarly, we update $\gamma_{\mathcal{E}}^{(\eta+1)}$ through solving

$$
\frac{\partial}{\partial \gamma_{\mathcal{E}}}\left[\Lambda_{2}^{(\eta)}\left(\gamma_{\mathcal{E}}, \boldsymbol{x}^{(\eta)}\right)\right]=0
$$

and update the MS position by

$$
\boldsymbol{x}^{(\eta+1)}=\underset{\boldsymbol{x}}{\arg \min } f_{2}(\boldsymbol{x}) .
$$

The expression of $f_{2}(\boldsymbol{x})$ and the results of the parameter update are given in Table I. Note that the conditional summations in the expressions of $\gamma_{\mathcal{E}}^{(\eta+1)}$ and $f_{2}(\boldsymbol{x})$ stem from the definition of $\mathcal{E}\left(v ; \gamma_{\mathcal{E}}\right)$, where the PDF is equal to zero when $v<0$.

Example-III: In the third example, we model the NLOS measurement errors by a Rayleigh distribution given by

$$
\mathcal{R}\left(v ; \gamma_{\mathcal{R}}\right)=\left\{\begin{array}{ll}
\frac{v}{\gamma_{\mathcal{R}}^{2}} \exp \left(\frac{-v^{2}}{2 \gamma_{\mathcal{R}}^{2}}\right), & v \geq 0 \\
0, & v<0
\end{array} .\right.
$$

The distribution parameter $\gamma_{\mathcal{R}}$ is strictly larger than zero. The parameter update process is very similar to the second example. Due to space limitations, we only show the final expressions in Table I.
For clarity, we summarize the main results of the given examples in Algorithm 1. The convergence conditions for the proposed algorithms follow the results to be given in Section V-B. Finally, we end up this subsection with the following remarks:

Remark 5: The ideal MLE, given precise propagation channel states in (6), can be solved iteratively using the same methodology shown in our examples. For the special case that the measurement error distributions (LOS and NLOS) are both Gaussian, we additionally show its parameter update on the $(\eta+1)$ th iteration in Table I for comparison purposes.

Remark 6: Comparing the parameter update (apart from $\alpha_{l}$ ) in Example-I with that in the ideal case, we can immediately find that the JMAP-ML estimator is equivalent to the ideal MLE for perfectly reconstructed the channel states $\mathbf{y}^{(\eta+1)}$. Whereas, the EM estimator always deviates from the ideal MLE as the weighting factors can be any value between zero and one.

Remark 7: Clearly, the two algorithms are simpler to implement as compared to the regular ML implementation of (9) in the given examples, since only the geographical coordinates have to be solved numerically and effective methods exist. 
Algorithm 1 EM- and JMAP-ML Algorithms for Joint Parameter Estimation using Range Measurements

\section{Step 1-Initialization:}

Choose a convergence tolerance $\Delta$ and the maximum number of iterations $N_{i t r}^{\max }$; Set the iteration index $\eta=0$; Choose an initial guess $\boldsymbol{\theta}^{(0)}=\left[\boldsymbol{\theta}_{m}^{(0)}, \boldsymbol{x}^{(0)}\right]^{T}$.

\section{Step 2-Joint Estimation:}

In the $(\eta+1)$ th iteration $(\eta \in \mathbb{Z}, \eta \geq 0)$,

- Compute $w_{i, k, l}^{(\eta)}$ according to (25) for $i=1,2, \ldots, N, k=$ $1,2, \ldots, K$, and $l=1,2$.

- Find closed form $\boldsymbol{\theta}_{m}^{(\eta+1)}$ in attempts to maximize $\Lambda^{(\eta)}\left(\boldsymbol{\theta}_{m}, \boldsymbol{x}^{(\eta)}\right)$.

- Find $\boldsymbol{x}^{(\eta+1)}=\left[x^{(\eta+1)}, y^{(\eta+1)}\right]^{T}$ in attempts to maximize $\Lambda^{(\eta)}\left(\boldsymbol{\theta}_{m}^{(\eta+1)}, \boldsymbol{x}\right)$ via numerical methods.

Step 3-Convergence Check:

If the increment of the log-likelihood value is less than $\Delta$ or $N_{i t r}^{\max }$ has been reached, then terminate this algorithm; otherwise set $\eta \leftarrow$ $\eta+1$ and return to Step 2 .

\section{B. Convergence Analysis}

As it is shown in Algorithm 1, both the EM algorithm and the JMAP-ML algorithm are iterative in nature. One question thus naturally arises: are the two algorithms ensured to converge? To answer this question, the following corollaries are derived.

Corollary 1: The proposed EM algorithm converges monotonically to some stationary point $\mathcal{L}_{1}^{*}{ }^{3}$ of the incomplete data log-likelihood function $\mathcal{L}_{I}(\boldsymbol{\theta} ; \mathbf{r})$.

Proof: In order to prove this corollary, we need to show in the first place that

$$
Q\left(\boldsymbol{\theta}^{(\eta+1)} ; \boldsymbol{\theta}^{(\eta)}\right) \geq Q\left(\boldsymbol{\theta}^{(\eta)} ; \boldsymbol{\theta}^{(\eta)}\right)
$$

holds for any $\boldsymbol{\theta}^{(\eta)}$ in its parameter space. The proof is as follows. For a given a priori position estimate $\boldsymbol{x}^{(\eta)}$, it is easy to show (we refer the readers to [27], [28]) that the updates $\alpha_{1}^{(\eta+1)}, \alpha_{2}^{(\eta+1)}, \boldsymbol{\beta}_{1}^{(\eta+1)}=\left[\mu_{1}^{(\eta+1)}, \sigma_{1}^{2,(\eta+1)}\right]^{T}, \boldsymbol{\beta}_{2}^{(\eta+1)}=$ $\left[\mu_{2}^{(\eta+1)}, \sigma_{2}^{2,(\eta+1)}\right]^{T}$ for the Gaussian distribution or $\boldsymbol{\beta}_{2}^{(\eta+1)}=$ $\gamma_{\mathcal{E}}^{(\eta+1)}$ for the exponential distribution or $\boldsymbol{\beta}_{2}^{(\eta+1)}=\gamma_{\mathcal{R}}^{(\eta+1)}$ for the Rayleigh distribution are global optimal solutions to the corresponding maximization problems. Therefore, we can easily conclude that

$$
Q\left(\boldsymbol{\theta}_{m}^{(\eta+1)}, \boldsymbol{x}^{(\eta)} ; \boldsymbol{\theta}^{(\eta)}\right) \geq Q\left(\boldsymbol{\theta}_{m}^{(\eta)}, \boldsymbol{x}^{(\eta)} ; \boldsymbol{\theta}^{(\eta)}\right)
$$

where the right-hand side is exactly $Q\left(\boldsymbol{\theta}^{(\eta)} ; \boldsymbol{\theta}^{(\eta)}\right)$.

We find a new position estimate $x^{(\eta+1)}$ by minimizing $f_{i \in\{1,2,3\}}(\boldsymbol{x})$ with respect to $\boldsymbol{x}$ via the BFGS quasi-Newton method with the initial guess set by $\boldsymbol{x}^{(\eta)}$. As mentioned beforehand, it guarantees downhill progress towards the local minimum in each Newton step, thus we conclude that the new position estimate $\boldsymbol{x}^{(\eta+1)}$ will not decrease $Q\left(\boldsymbol{\theta} ; \boldsymbol{\theta}^{(\eta)}\right)$ on the $(\eta+1)$ th iteration, more specifically,

$$
Q\left(\boldsymbol{\theta}_{m}^{(\eta+1)}, \boldsymbol{x}^{(\eta+1)} ; \boldsymbol{\theta}^{(\eta)}\right) \geq Q\left(\boldsymbol{\theta}_{m}^{(\eta+1)}, \boldsymbol{x}^{(\eta)} ; \boldsymbol{\theta}^{(\eta)}\right)
$$

\footnotetext{
${ }^{3}$ In this paper, a stationary point could be a saddle point, a local optimum or a global optimum.
}

where the left-hand side is identical to $Q\left(\boldsymbol{\theta}^{(\eta+1)} ; \boldsymbol{\theta}^{(\eta)}\right)$. Combining the results in (43) and (44), we complete the proof of (42). Since $\mathcal{L}\left(\boldsymbol{\theta}^{(\eta)} ; \mathbf{r}\right)$ is clearly bounded from above, a direct application of [14, Theorem 1] in the next step will prove our corollary.

Corollary 2: The proposed JMAP-ML algorithm converges monotonically to some stationary point $\mathcal{L}_{2}^{*}$ of the complete data log-likelihood function $\mathcal{L}_{C}(\boldsymbol{\theta} ; \mathbf{y}, \mathbf{r})$.

Proof: In the first step of the JMAP-ML algorithm, we maximize $\mathcal{L}(\boldsymbol{\theta} ; \mathbf{y}, \mathbf{r})$ with respect to $\mathbf{y}$ for a given a priori parameter estimate $\boldsymbol{\theta}^{(\eta)}$. Since $\mathbf{y}^{(\eta+1)}$ is the global optimal solution, it is guaranteed that

$$
\mathcal{L}_{C}\left(\boldsymbol{\theta}^{(\eta)} ; \mathbf{y}^{(\eta+1)}, \mathbf{r}\right) \geq \mathcal{L}_{C}\left(\boldsymbol{\theta}^{(\eta)} ; \mathbf{y}^{(\eta)}, \mathbf{r}\right)
$$

holds for any $\mathbf{y}^{(\eta)}$ in its parameter space. In the second step, we maximize $\mathcal{L}\left(\boldsymbol{\theta} ; \mathbf{y}^{(\eta+1)}, \mathbf{r}\right)$ with respect to $\boldsymbol{\theta}$. Following the same procedure for proving $Q\left(\boldsymbol{\theta}^{(\eta+1)} ; \boldsymbol{\theta}^{(\eta)}\right) \geq Q\left(\boldsymbol{\theta}^{(\eta)} ; \boldsymbol{\theta}^{(\eta)}\right)$ in Corollary 1 , we can easily prove that

$$
\mathcal{L}_{C}\left(\boldsymbol{\theta}^{(\eta+1)} ; \mathbf{y}^{(\eta+1)}, \mathbf{r}\right) \geq \mathcal{L}_{C}\left(\boldsymbol{\theta}^{(\eta)} ; \mathbf{y}^{(\eta+1)}, \mathbf{r}\right)
$$

which means that the value of $\mathcal{L}(\boldsymbol{\theta} ; \mathbf{y}, \mathbf{r})$ increases monotonically over iterations. Since $\mathcal{L}(\boldsymbol{\theta} ; \mathbf{y}, \mathbf{r})$ is bounded from above, the convergence to some stationary point $\mathcal{L}_{2}^{*}$ of $\mathcal{L}_{C}(\boldsymbol{\theta} ; \mathbf{y}, \mathbf{r})$ is ensured.

\section{Complexity Analysis}

In the complexity analysis, we spotlight the evaluation of the joint estimation step (the second step of the above algorithms), as it consumes the most computational power. The complexity is evaluated in terms of floating-point operations (FLOPs). Due to space limitations, we only show the details of this analysis in Appendix A for the EM algorithm in the GaussianGaussian mixture case. Therein, it is shown that the EM algorithm requires $\mathcal{O}(N K)$ FLOPs to compute an estimate. The computation of a JMAP-ML estimate is very similar to that of an EM estimate. Hence, it is easy to verify that the complexity of the JMAP-ML algorithm is of order $\mathcal{O}(N K)$ FLOPs as well. The complexity analysis of the RIN algorithm can be performed in the same manner, and it needs $\mathcal{O}\left((N K)^{2}\right)$ FLOPs to compute a position estimate. The same conclusion can be drawn for the other two examples given in section $\mathrm{V}-\mathrm{A}$.

\section{Cramér-RAo Lower Bound COMPutation}

It is well known that the covariance matrix of any unbiased estimator of an unknown vector parameter is lower bounded by the Cramér-Rao lower bound [11]. In the sequel, a numerical method is proposed to compute the CRLB for our joint estimation problem. The vector parameter to be determined is $\boldsymbol{\theta} .{ }^{4}$ Let $\hat{\boldsymbol{\theta}}$ be an unbiased estimator of $\boldsymbol{\theta}$ and let $\operatorname{Cov}(\hat{\boldsymbol{\theta}})$ denote the covariance matrix of $\hat{\boldsymbol{\theta}}$. Assuming that certain regularity conditions are fulfilled [29, C.2], we have

$$
\begin{aligned}
\operatorname{Cov}(\hat{\boldsymbol{\theta}}) & =\mathbb{E}_{p(\mathbf{r} ; \boldsymbol{\theta})}\left\{(\hat{\boldsymbol{\theta}}-\boldsymbol{\theta})(\hat{\boldsymbol{\theta}}-\boldsymbol{\theta})^{T}\right\} \\
& \succeq \mathcal{F}^{-1}(\boldsymbol{\theta}) \equiv \mathrm{CRLB}
\end{aligned}
$$

${ }^{4}$ Note that $\boldsymbol{\theta}=\left[\boldsymbol{\beta}_{1}^{T}, \boldsymbol{\beta}_{2}^{T}, \boldsymbol{x}^{T}\right]^{T}$ in (6) or $\boldsymbol{\theta}=\left[\alpha_{1}, \alpha_{2}, \boldsymbol{\beta}_{1}^{T}, \boldsymbol{\beta}_{2}^{T}, \boldsymbol{x}^{T}\right]^{T}$ in 
where $\mathcal{F}(\boldsymbol{\theta})$ denotes Fisher information matrix (FIM).

The FIM can be expressed as

$$
\mathcal{F}(\boldsymbol{\theta})=\mathbb{E}_{p(\mathbf{r} ; \boldsymbol{\theta})}\left\{-\Delta_{\boldsymbol{\theta}}^{\boldsymbol{\theta}} \ln p(\mathbf{r} ; \boldsymbol{\theta})\right\}
$$

where the expectation is taken with respect to $p(\mathbf{r} ; \boldsymbol{\theta})$, namely the PDF of $\mathbf{r}$ parameterized by $\boldsymbol{\theta}$. Often, it is more convenient to express the FIM as follows:

$$
\begin{aligned}
\mathcal{F}(\boldsymbol{\theta}) & =\mathbb{E}_{p(\mathbf{r} ; \boldsymbol{\theta})}\left\{\nabla_{\boldsymbol{\theta}} \ln p(\mathbf{r} ; \boldsymbol{\theta}) \cdot \nabla_{\boldsymbol{\theta}}^{T} \ln p(\mathbf{r} ; \boldsymbol{\theta})\right\} \\
& =\int\left\{\nabla_{\boldsymbol{\theta}} \ln p(\mathbf{r} ; \boldsymbol{\theta}) \cdot \nabla_{\boldsymbol{\theta}}^{T} \ln p(\mathbf{r} ; \boldsymbol{\theta})\right\} p(\mathbf{r} ; \boldsymbol{\theta}) \mathrm{d} \mathbf{r} .
\end{aligned}
$$

Under the mixture density assumption, the integration in (49) is hard to evaluate analytically. In order to give a numerical solution, we perform Monte Carlo integration [30] as follows:

$$
\mathcal{F}(\boldsymbol{\theta}) \approx \frac{1}{N_{M}} \sum_{n=1}^{N_{M}}\left\{\nabla_{\boldsymbol{\theta}} \ln p\left(\mathbf{r}^{(n)} ; \boldsymbol{\theta}\right) \cdot \nabla_{\boldsymbol{\theta}}^{T} \ln p\left(\mathbf{r}^{(n)} ; \boldsymbol{\theta}\right)\right\}
$$

where $\mathbf{r}^{(n)}, n=1,2, \ldots, N_{M}$ are sample vectors generated independently from $p(\mathbf{r} ; \boldsymbol{\theta}) .^{5}$ The calculation of $\nabla_{\boldsymbol{\theta}} \ln p(\mathbf{r} ; \boldsymbol{\theta})$ is straightforward for the examples shown in Section V-A and thus not shown here due to space limitations. However, we have to be careful with the regularity conditions [29, C.2]. Violation of any regularity condition may result in unwanted results.

In this paper, we focused on the performance of the position estimator $\hat{\boldsymbol{x}}=[\hat{x}, \hat{y}]^{T}$ amid $\hat{\boldsymbol{\theta}}$. Serving as localization accuracy in practice, the positioning root mean square error (RMSE) is considered here to evaluate different position estimators. As it is well known, the RMSE of an unbiased position estimator is defined by

$$
\mathrm{RMSE} \equiv \sqrt{\mathbb{E}_{p(\mathbf{r} ; \boldsymbol{\theta})}\left\{(\hat{x}-x)^{2}+(\hat{y}-y)^{2}\right\}}
$$

and it is related to the obtained CRLB according to

$$
\operatorname{RMSE} \geq \sqrt{\left(\left[\mathcal{F}^{-1}(\boldsymbol{\theta})\right]_{q-1, q-1}+\left[\mathcal{F}^{-1}(\boldsymbol{\theta})\right]_{q, q}\right)} \equiv \mathrm{CRLB}_{\mathrm{pos}}
$$

where $q \triangleq \operatorname{dim}(\boldsymbol{\theta})$ denotes the dimension of $\boldsymbol{\theta}$, and CRLB $\mathrm{Bos}_{\mathrm{p}}$ is defined to interpret the best achievable localization accuracy of any unbiased position estimator.

\section{Simulations}

We consider a specific geolocation problem in a cellular radio network comprising $N=4$ BSs. The positions of the BSs are obtained from an operating cellular radio network in a German city and they are depicted in Fig. 1. Gaussian ditribution is assumed for the measurement error under both LOS and NLOS conditions. The measurement error model parameters are set according to the measurement campaigns

\footnotetext{
${ }^{5}$ Here, $\ln (p(\mathbf{r} ; \boldsymbol{\theta}))$ follows the expression in (6) for the ideal case or the expression in (8) for the approximated case. In the former case, we generate $\mathbf{v}^{(n)}=\left[\mathbf{v}_{1}^{(n)}, \ldots, \mathbf{v}_{N}^{(n)}\right]$ in the $n$th Monte Carlo run, where $\mathbf{v}_{i}^{(n)}$ contains $K$ samples generated either from $p_{V}^{(1)}\left(v ; \boldsymbol{\beta}_{1}\right)$ or $p_{V}^{(2)}\left(v ; \boldsymbol{\beta}_{1}\right)$ depending on the channel state. A sample vector $\mathbf{r}^{(n)}$ is immediately obtained owing to the relationship $\mathbf{r}^{(n)}=\mathbf{h}(\boldsymbol{x})+\mathbf{v}^{(n)}$ as defined in (2). In the latter case, $\mathbf{v}^{(n)}$ contains $N K$ measurement error samples that are generated independently and identically from $p_{V}(v)=\alpha_{1} p_{V}^{(1)}\left(v ; \boldsymbol{\beta}_{1}\right)+\alpha_{2} p_{V}^{(2)}\left(v ; \boldsymbol{\beta}_{2}\right)$.
}

TABLE II

Simulation PARAMETERS

\begin{tabular}{|l|l|}
\hline Parameter & Value \\
\hline$N$ & 4 \\
\hline$\mu_{1}$ & 0 meter $(\mathrm{m})$ \\
\hline$\sigma_{1}$ & $55 \mathrm{~m}$ \\
\hline$\mu_{2}$ & $380 \mathrm{~m}$ \\
\hline$\sigma_{2}$ & $120 \mathrm{~m}$ \\
\hline$x$ & $250 \mathrm{~m}$ \\
\hline$y$ & $250 \mathrm{~m}$ \\
\hline$\Delta$ & $10^{-6}$ \\
\hline$N_{i t r}^{\max }$ & 40 iterations \\
\hline$N_{M}$ & 50,000 samples \\
\hline
\end{tabular}

conducted in the same city [10]. It is noteworthy to mention that we reset $\mu_{1}$ from 51 meter ${ }^{6}$ to 0 meter and assume it to be known in order to comply with the common assumption that the measurement error in LOS channel is unbiased. In Table II, we summarize the simulation parameters that will be used throughout this section. Based on these parameters, we generate simulated data to test different algorithms.

The newly proposed estimators are compared to the following competitors:

- Robust iterative nonparametric (RIN) position estimator, cf. [13, Algorithm 1].

- Generic identify and discard (IAD)-ML estimator. The gist of the IAD based algorithms is to identify and discard the NLOS measurements and use the LOS measurements only for geolocation. As we assume very limited prior knowledge about the measurement error statistics, the identification step of the existing algorithms, e.g., those in [23], [31], is either infeasible or fails to generate reliable performance. For comparison purposes only, we consider two simplified cases. In the first case, we ideally assume both the LOS channels and the NLOS channels are precisely categorized in every Monte-Carlo run. In the second case, we assume one NLOS channel is wrongly recognized as LOS channel for 5\% of the total MonteCarlo runs. Like in [32], the remaining LOS measurements are used in the maximum-likelihood estimation of the position.

- Ideal maximum-likelihood estimator, given the precise prior knowledge about the channel states (cf. (6)). This ideal estimator is computed in an iterative process as described in section $\mathrm{V}$-A.

Moreover, we compare the positioning RMSE of different estimators with the best achievable localization accuracy computed by (52) with the log-likelihood function (6).

The initial guess of the MS position for the above algorithms is equal to the true value contaminated by $\boldsymbol{\Delta}_{x y}$ whose elements are generated from a uniform distribution $\mathcal{U}(-50,50)$ (in meter). The initial guess of the mixture model parameters for the JMAP-ML algorithm and the EM algorithm is computed according to Algorithm 2, given in Appendix B. All the simulations considered afterwards are performed in a MATLAB ${ }^{\mathrm{TM}} \mathrm{R} 2010$ a environment.

\footnotetext{
${ }^{6}$ The positive mean in the LOS component from the field trial might be a result of quantization.
} 


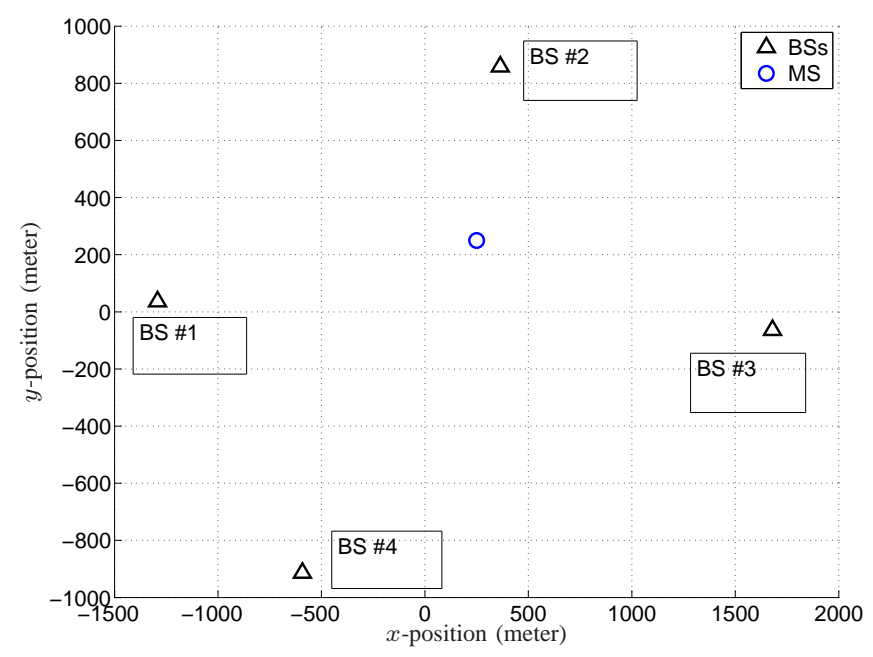

Fig. 1. 2-D illustration of the geometry of BSs in an operating cellular radio network in Germany.

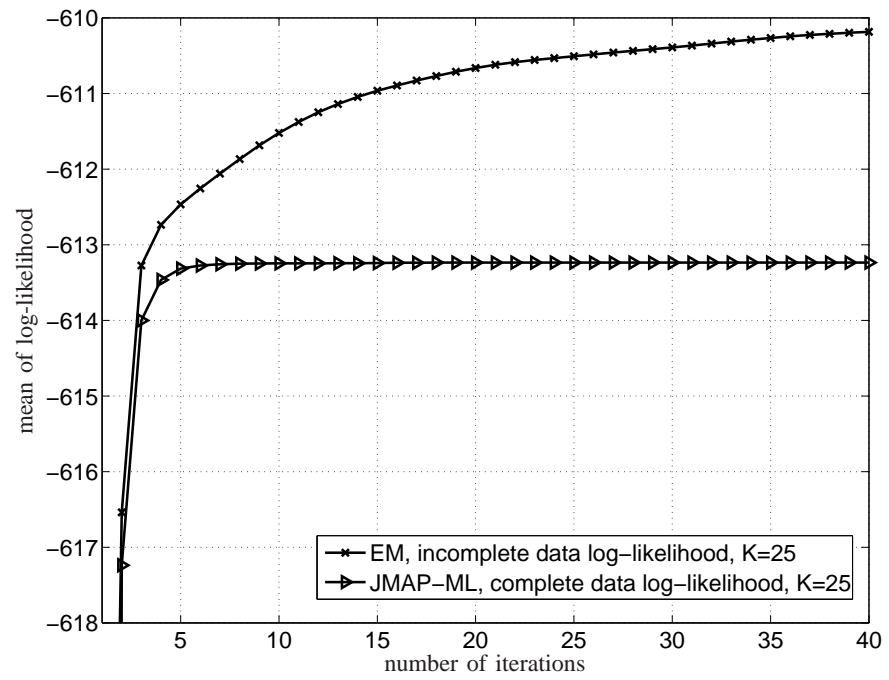

Fig. 2. Convergence of the mean of the log-likelihood versus the number of iterations for both the EM algorithm and the JMAP-ML algorithm.

\section{A. Convergence Properties}

In the first experiment, we numerically study the convergence properties of the newly proposed iterative algorithms. Here, we assume that the second BS is a NLOS BS while the rest are LOS BSs. The number of measurements collected at each BS is set to $K=25$ samples.

In order to experimentally assess Corollary 1 and Corollary 2, we perform a Monte Carlo experiment with 1500 independent runs. For each Monte-Carlo run, we record the parameter estimates and the corresponding data log-likelihoods ${ }^{7}$ versus the number of iterations for both the EM algorithm and the JMAP-ML algorithm. Thereafter, we compute the mean of the parameter estimates as well as the mean of the data loglikelihoods, respectively. The mean of the data log-likelihoods

\footnotetext{
${ }^{7}$ It is necessary to stress here again that we consider the incomplete data log-likelihood for the EM algorithm whereas consider the complete data loglikelihood for the JMAP-ML algorithm.
}
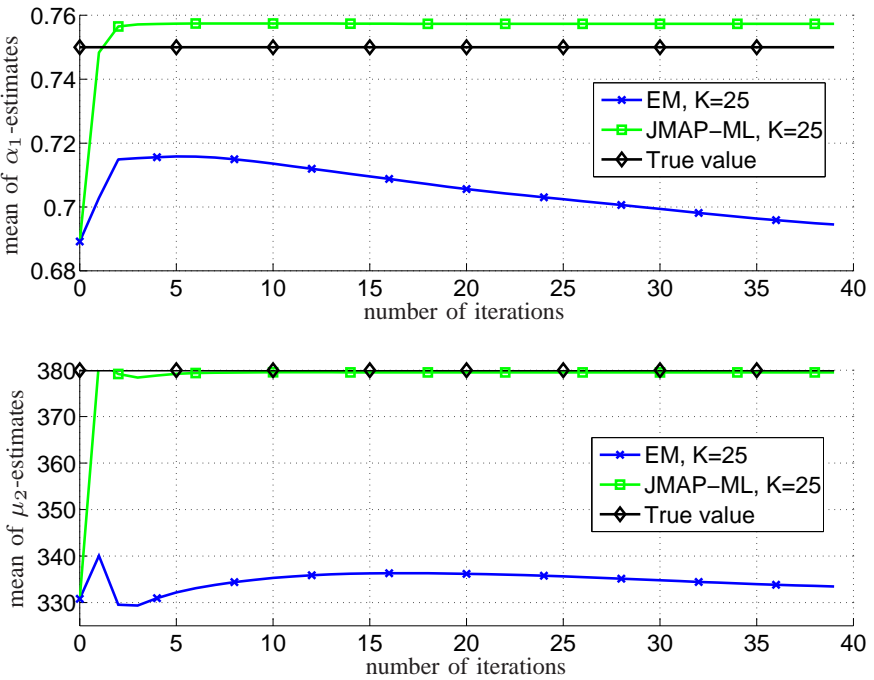

Fig. 3. Top: Mean of $\alpha_{1}$ estimates versus the number of iterations. Bottom: Mean of $\mu_{2}$ estimates versus the number of iterations.
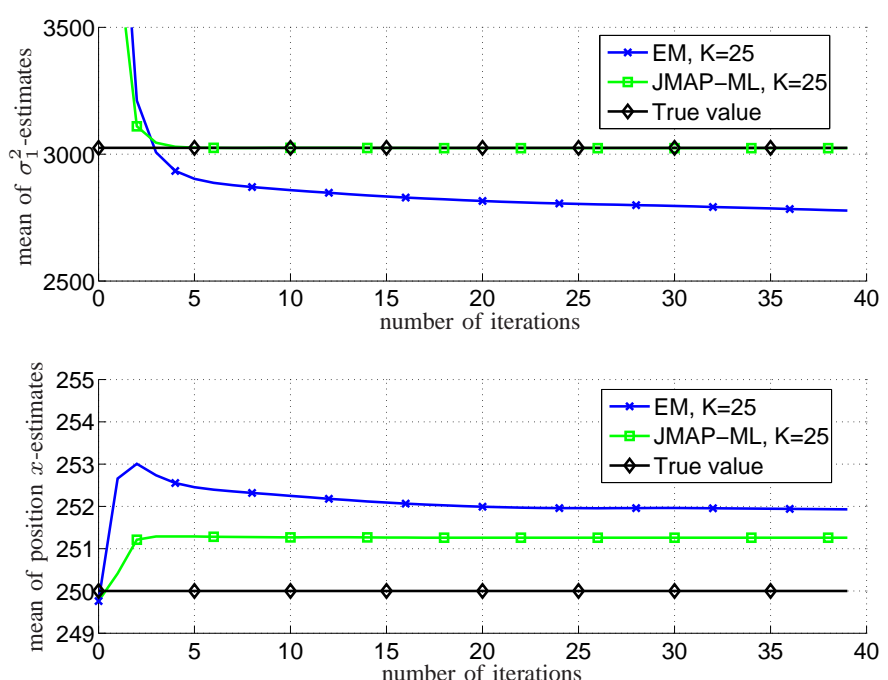

Fig. 4. Top: Mean of $\sigma_{1}^{2}$ estimates versus the number of iterations. Bottom: Mean of position $x$ estimates versus the number of iterations.

is shown versus the number of iterations in Fig. 2. Due to space limitations, we only show the mean of the parameter estimates for $\alpha_{1}, \mu_{2}, \sigma_{1}^{2}$, and $x$ in Fig. 3 and Fig. 4. For better illustration, the true values of the desired parameters are also depicted.

Next, we summarize some important observations and give the relevant explanations as follows:

- Figure 2 shows that the two data log-likelihoods increase monotonically over iterations until the convergence condition has been fulfilled, which coincides with our theoretical proofs.

- Both the EM algorithm and the JMAP-ML algorithm generates biased estimator. The JMAP-ML estimator is biased in itself, but in this example the resulting bias is nearly negligible. On the contrary, the EM estimator shows larger bias. This is due to the mismatch of the underlying measurement error statistics and the assumed 


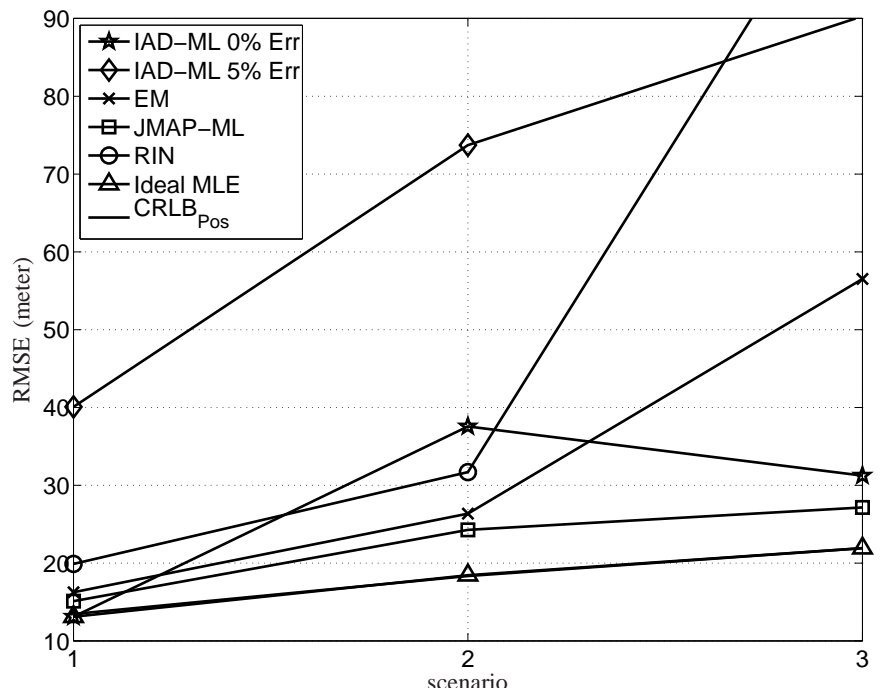

Fig. 5. Positioning RMSE of different position estimators in three different scenarios.

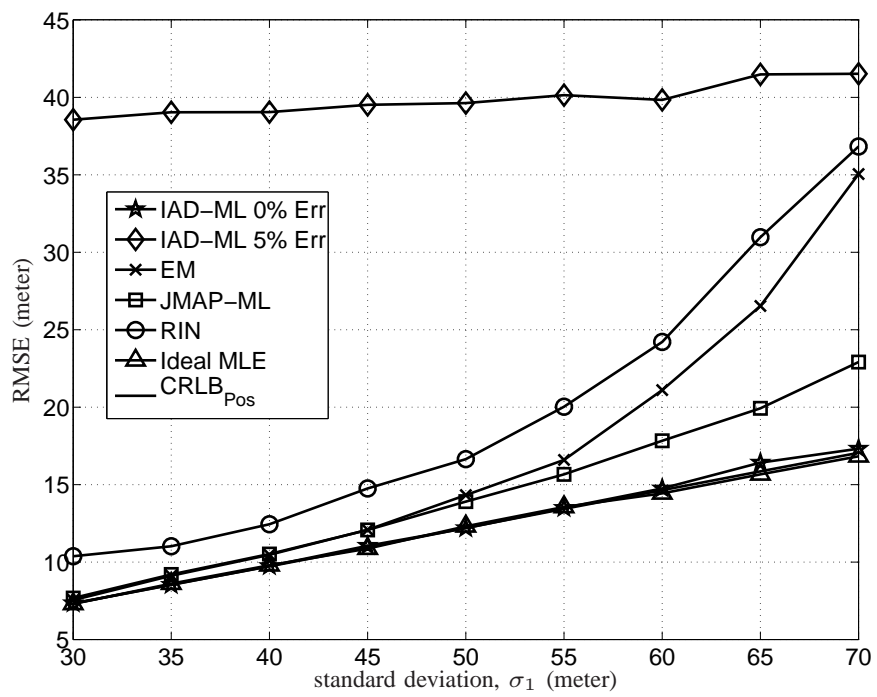

Fig. 6. Positioning RMSE of different position estimators versus the LOS measurement error standard deviation $\sigma_{1}$

one.

- Given the same initial guess, the JMAP-ML algorithm outperforms the EM algorithm in terms of convergence speed.

\section{B. Localization Accuracy}

In the second experiment, we shall investigate the localization performance (in terms of the positioning RMSE) for different estimators. The simulation parameters remain unaltered, unless otherwise specified.

The first example assumes three different geolocation scenarios: (1) \{LOS BS\#1, LOS BS\#2, LOS BS\#3, NLOS BS\#4\}, (2) $\{$ NLOS BS\#1, LOS BS\#2, NLOS BS\#3, LOS BS\#4\}, and (3) $\{$ LOS BS\#1, NLOS BS\#2, NLOS BS\#3, NLOS BS\#4\}. For each scenario, we evaluate the positioning RMSE for different estimators. The results are shown in Fig. 5. In the

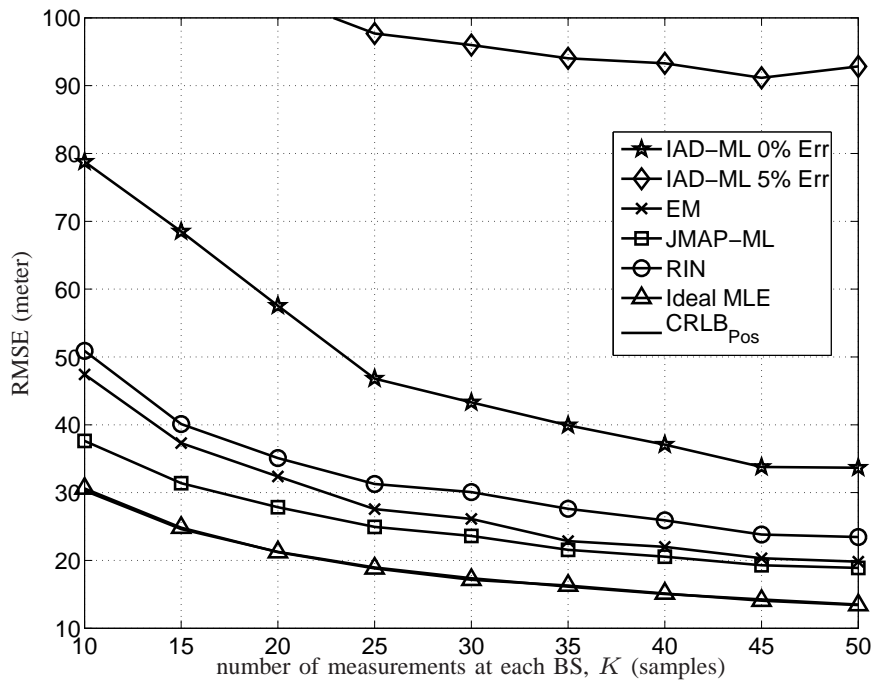

Fig. 7. Positioning RMSE of different position estimators versus the number of measurements $K$ at each of $N=4$ BSs.

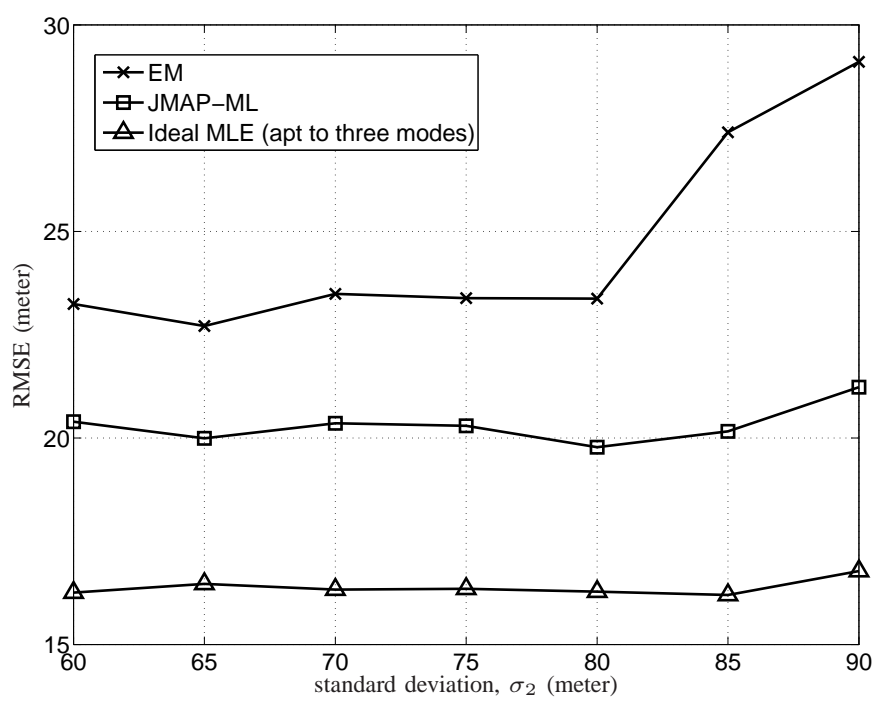

Fig. 8. Positioning RMSE of different position estimators versus the standard deviation $\sigma_{2}$.

second example, we study the influence of $\sigma_{1}$ on the positioning RMSE. Herein, we focus on the $\{$ LOS BS\#1, LOS BS\#2, LOS BS\#3, NLOS BS\#4\} scenario and increase $\sigma_{1}$ from 30 to 70 meter. The results are shown in Fig. 6. In the third example, we investigate how the number of measurements $K$ affects the RMSE curves. For this example, we consider the $\{$ LOS BS\#1, NLOS BS\#2, LOS BS\#3, NLOS BS\#4\} scenario and vary $K$ from 10 to 50 samples. The RMSE curves are depicted in Fig. 7. In the above examples, the best achievable localization accuracy $\mathrm{CRLB}_{\text {pos }}$ is also computed and depicted along with the RMSE curves.

From the illustrations, we summarize the performance of the selected estimators as follows:

- The ideal MLE is nearly unbiased (although not shown here) and attains the performance bound for large $K$. This coincides with the asymptotic property of the MLE in theory. 
- The JMAP-ML estimator is closest to the ideal MLE in terms of both the RMSE and the bias (cf. Fig. 3 and Fig. 4). This is because the knowledge of the actual channel states y is mostly well reconstructed.

- The EM estimator is biased as well and provides inferior RMSE as compared to that of the JMAP-ML estimator. But still, it serves as a good approximation of the ideal MLE in many cases.

- The EM estimator always outperforms the RIN estimator. The reason is that it possesses more information about the measurement error than the RIN algorithm.

- In some cases, both the EM algorithm and the RIN algorithm break down. The key reason is that the cost function for updating the position deviates too much from the ideal one. To remedy the performance loss of the RIN algorithm, we could run AKDE individually at each BS.

- The IAD-ML estimator (with $5 \%$ erroneous identification rate) performs the worst due to the outliers (the survival NLOS measurements). The performance will further deteriorate as the erroneous identification rate increases.

- The IAD-ML estimator (with $0 \%$ erroneous identification rate) can even achieve almost the same performance as the ideal one in some cases. Possible reasons are the following. First, the number of remaining LOS BSs is still sufficient (larger or equal to three). Secondly, after discarding the NLOS measurements the geometric dilution of precision (GDOP) is improved. Thirdly, although we assume known distribution for the NLOS measurement error, the associated parameters are unknown and the variance is set large. Hence, the information about the position hidden in the NLOS measurements is negligible. As is known from [32], NLOS measurements can be discarded without losing any information when the noise variance goes to infinity. Fourthly, we have more parameters to estimate for the proposed algorithms as compared to the IAD-ML algorithm (only the position needs to be estimated according to our simulation setup).

- The IAD-ML estimator (with $0 \%$ erroneous identification rate) degrades largely when the number of LOS BSs is insufficient for geolocation after discarding the detected NLOS BSs.

In the third experiment, we consider another model mismatch problem and give a glimpse of its impact on the localization accuracy. Here, we consider a scenario \{LOS BS\#1, LOS BS\#2, NLOS BS\#3, NLOS BS\#4\}. In contrast to the above experiments, we assume the measurement error distributions are different for the third and the fourth NLOS BSs. More precisely, we assume $\mu_{1}=0, \sigma_{1}=55$ for the first two LOS BSs, and $\mu_{2}=20, \sigma_{2}=60: 5: 90$ for the third NLOS BS, and $\mu_{3}=380, \sigma_{3}=120$ for the fourth NLOS BS. We generate simulated data based on the new parameter settings but still apply the EM algorithm and the JMAP-ML algorithm developed intentionally for a two-mode mixture. Serving as the benchmark algorithm, the ideal MLE is adapted to the new settings. The positioning RMSE curves are drawn as a function of $\sigma_{2}$ in Fig. 8. In this experiment, only moderate performance loss has been observed. The reason

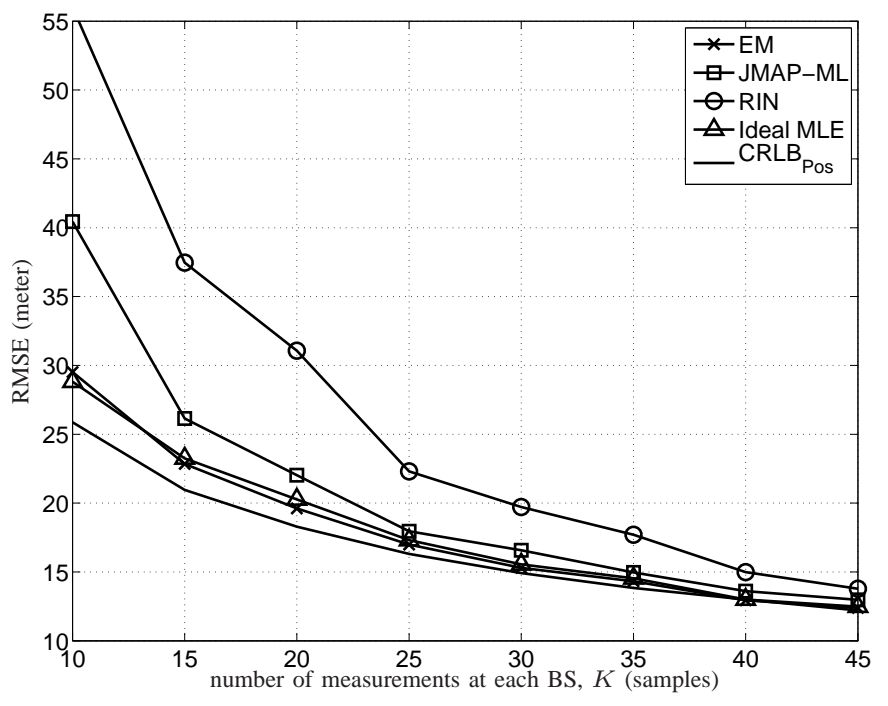

Fig. 9. Positioning RMSE of different position estimators versus the number of measurements $K$ at each of $N=4$ BSs. In contrast to Fig. 7, the measurement errors are independently and identically (although unrealistic) generated from $p_{V}(v)=\alpha_{1} p_{V}^{(1)}\left(v ; \boldsymbol{\beta}_{1}\right)+\alpha_{2} p_{V}^{(2)}\left(v ; \boldsymbol{\beta}_{2}\right)$ in this experiment.

is that the proposed algorithms (assuming $l=2$ ) can always arrive at a two-mode Gaussian mixture that well approximates the "truth". However, we believe that this mismatch problem may incur more severe performance loss in certain cases. A comprehensive study of this issue is beyond the scope of this paper.

\section{Under the i.i.d. Assumption}

In our geolocation problem, we suboptimally represent the measurement error with a two-mode mixture distribution to trade the desired properties of the proposed algorithms. As we have seen in the previous experiments, the performance loss due to the model mismatch exits but turns out to be moderate. In some real geolocation applications, the underlying measurement error can well match a mixture distribution. For instance, the application of the single moving sensor geolocation [33, Chapter 17]. Besides, there may exist other applications, cf. $[13, \mathrm{VI}-\mathrm{G}]$, where the measurement error samples are truly i.i.d. and follow a mixture distribution. Our aim in the last experiment is to shed some light on the performance of the proposed algorithms assuming no model mismatch. Although unrealistic, we redo the the third example of the second experiment by assuming the measurement error items are i.i.d. and follow a two-mode Gaussian distribution with $\alpha_{1}=\alpha_{2}=0.5$. We plot the RMSE curves of the EM estimator, the JMAPML estimator, the RIN estimator, and the ideal MLE along with the performance lower bound in Fig. 9. Note that the ideal MLE in this example is the solution of (9), which is resolved via the MATLAB ${ }^{\text {TM }}$ function FMINCON, configured with the "interior-point" algorithm. In order to increase the probability of hitting the global maximum, we employ several different initial guesses in the vicinity of the true value of $\boldsymbol{\theta}$ and choose the one maximizing the cost function. Although the global maximum is not guaranteed to be found by a local search algorithm in every Monte Carlo trial, we believe that 
this numerical solution well reveals the ground truth in most cases. From Fig. 9, we found that the EM algorithm is able to reproduce the ideal MLE and outperforms both the JMAPML algrithm and the RIN algorithm. Moreover, all the three algorithms tend to achive the performance lower bound for large data records.

\section{CONCLUSION}

We presented here an EM algorithm and a JMAP-ML algorithm for robust geolocation in mixed LOS/NLOS environments. In the algorithm design, we suboptimally represent the underlying measurement error with a two-mode mixture distribution. In return, the proposed algorithms avoid the offline calibration and require no prior knowledge about the exact channel states. Although starting from different design criteria, the proposed algorithms arrive at similar maximization problems, from which the parameter estimates are updated correspondingly. The proposed algorithms are both iterative in nature, and we demonstrated their convergence properties along with examples. Therein, we also proved that the complexity of the two algorithms increases linearly with the number of measurements $N K$. Despite the model mismatch problem, the JMAP-ML estimator well approximates the ideal MLE in our simulations. Although the EM algorithm loses its optimality, it still outperforms various competitors by far. In the applications where the underlying measurement error exactly fits our assumed model, both the EM algorithm and the JMAP-ML algorithm tend to achieve the performance bound for large data records.

\section{APPENDIX A}

\section{COMPUTATIONAL COMPLEXITY OF THE EM AlgorithM: Gaussian-Gaussian MiXture Case}

We define the FLOPs required for some elementary operations as follows:

1) $\mathcal{E}_{\text {add }}$ : FLOPs for addition.

2) $\mathcal{E}_{s u b}$ :FLOPs for substraction.

3) $\mathcal{E}_{m u l}$ : FLOPs for multiplication.

4) $\mathcal{E}_{\text {div }}$ : FLOPs for division.

5) $\mathcal{E}_{\text {exp }}$ : FLOPs for exponential.

6) $\mathcal{E}_{\text {pow }}$ : FLOPs for raising to real power.

7) $\mathcal{E}_{\text {sqrt }}$ : FLOPs for square root.

Note that the actual FLOPs required for the above operations may vary with processors. Typical values based on a Pentium $囚 4$ processor were given in [34].

Since the EM algorithm is iterative in nature, we spotlight the analysis in one EM iteration, for instance the $(\eta+1)$ th. The joint estimation step starts with the evaluations of $w_{i, k, l}^{(\eta)}=$ $\tilde{P}_{i, k, l}^{(\eta)}$ for $i=1,2, \ldots, N, k=1,2, \ldots, K$ and $l=1,2$, given the prior parameter estimate $\boldsymbol{\theta}^{(\eta)}$. This requires us to compute

$$
\operatorname{Eval}(1): d_{i}\left(\boldsymbol{x}^{(\eta)}\right)=\sqrt{\left(x_{i}-x^{(\eta)}\right)^{2}+\left(y_{i}-y^{(\eta)}\right)^{2}}
$$

for $i=1,2, \ldots, N$.

$$
\operatorname{Eval}(2): r_{i, k}-d_{i}\left(\boldsymbol{x}^{(\eta)}\right)
$$

for $i=1,2, \ldots, N, k=1,2, \ldots, K$.

$$
\begin{aligned}
& \operatorname{Eval}(3): \\
& \Phi_{i, k, l}=\frac{\alpha_{l}^{(\eta)}}{\sqrt{2 \pi \sigma_{l}^{2,(\eta)}}} \cdot \exp \left[\frac{-\left(r_{i, k}-d_{i}\left(\boldsymbol{x}^{(\eta)}\right)-\mu_{l}^{(\eta)}\right)^{2}}{2 \sigma_{l}^{2,(\eta)}}\right]
\end{aligned}
$$

for $i=1,2, . ., N, k=1,2, \ldots, K, l=1,2$.

$$
\operatorname{Eval}(4): \tilde{P}_{i, k, l}^{(\eta)}=\frac{\Phi_{i, k, l}}{\sum_{l=1}^{2} \Phi_{i, k, l}}
$$

for $i=1,2, \ldots, N, k=1,2, \ldots, K, l=1,2$.

It is clear that $\operatorname{Eval}(1)$ requires $N\left(2 \mathcal{E}_{\text {sub }}+2 \mathcal{E}_{\text {pow }}+\right.$ $\left.1 \mathcal{E}_{\text {sqrt }}+1 \mathcal{E}_{\text {add }}\right)$ FLOPs, Eval $(2)$ requires $N K \mathcal{E}_{\text {sub }}$ FLOPs, $\operatorname{Eval}(3)$ requires $2\left((N K+3) \mathcal{E}_{\text {mul }}+(N K+1) \mathcal{E}_{\text {pow }}+\right.$ $\left.N K \mathcal{E}_{\text {sub }}+N K \mathcal{E}_{\text {div }}+N K \mathcal{E}_{\text {exp }}\right)$ FLOPs, and $\operatorname{Eval}(4)$ requires $N K\left(1 \mathcal{E}_{\text {sub }}+1 \mathcal{E}_{\text {add }}+1 \mathcal{E}_{\text {div }}\right)$ FLOPs.

Having $\tilde{P}_{i, k, l}^{(\eta)}$, s, we then compute

$$
\begin{aligned}
& \operatorname{Eval}(5): \alpha_{l}^{(\eta+1)}, l=1,2 . \\
& \operatorname{Eval}(6): \mu_{l}^{(\eta+1)}, l=1,2 . \\
& \operatorname{Eval}(7): \sigma_{l}^{2,(\eta+1)}, l=1,2 .
\end{aligned}
$$

It is easy to verify that $\operatorname{Eval}(5)$ requires $(N K-1) \mathcal{E}_{a d d}+$ $1 \mathcal{E}_{\text {div }}+1 \mathcal{E}_{\text {sub }}$ FLOPs, Eval $(6)$ requires $2\left(N K \mathcal{E}_{m u l}+(N K-\right.$ $\left.1) \mathcal{E}_{a d d}+\mathcal{E}_{\text {div }}\right)$ FLOPs, and $\operatorname{Eval}(7)$ requires $2((N K+$ 1) $\left.\mathcal{E}_{\text {pow }}+N K \mathcal{E}_{\text {mul }}+(N K-1) \mathcal{E}_{a d d}+\mathcal{E}_{d i v}+\mathcal{E}_{\text {sub }}\right)$ FLOPs. Let us define $F L\left(\boldsymbol{\theta}_{m}\right)$ to be the total number of FLOPs consumed for an estimate of $\boldsymbol{\theta}_{m}$ in one EM iteration. It is straightforward that $F L\left(\boldsymbol{\theta}_{m}\right)$ is equal to the total FLOPs consumed in $\operatorname{Eval}(1)$ through $\operatorname{Eval}(7)$, and the final result is

$$
\begin{array}{r}
F L\left(\boldsymbol{\theta}_{m}\right)=(6 N K-5) \mathcal{E}_{a d d}+(4 N K+N+1) \mathcal{E}_{\text {sub }} \\
+(6 N K+6) \mathcal{E}_{\text {mul }}+(3 N K+4) \mathcal{E}_{\text {div }}+N \mathcal{E}_{\text {sqrt }} \\
+(4 N K+2 N+4) \mathcal{E}_{\text {pow }}+2 N K \mathcal{E}_{\text {exp }} .
\end{array}
$$

The numerical evaluation of $\boldsymbol{x}^{(\eta+1)}$ attempts to minimize $f_{1}(\boldsymbol{x})$ via the BFGS quasi-Newton method which involves another iterative procedure similar to [13, Algorithm 2]. Analogously, we count the FLOPs required for one iteration of this local search. This requires constant evaluation of the cost function $f_{1}(\boldsymbol{x})$ and its gradient at a certain point $\boldsymbol{x}$, namely,

$$
\begin{aligned}
& \operatorname{Eval}(8): f_{1}(\boldsymbol{x})= \\
& \frac{1}{\sigma_{1}^{2,(\eta+1)}} \sum_{i=1}^{N} \sum_{k=1}^{K}\left(r_{i, k}-d_{i}(\boldsymbol{x})-\mu_{1}^{(\eta+1)}\right)^{2} \tilde{P}_{i, k, 1}^{(\eta)} \\
& +\frac{1}{\sigma_{2}^{2,(\eta+1)}} \sum_{i=1}^{N} \sum_{k=1}^{K}\left(r_{i, k}-d_{i}(\boldsymbol{x})-\mu_{2}^{(\eta+1)}\right)^{2} \tilde{P}_{i, k, 2}^{(\eta)} . \quad(6 \\
& \operatorname{Eval}(9): \nabla_{x} f_{1}(\boldsymbol{x})= \\
& \frac{2}{\sigma_{1}^{2,(\eta+1)}} \sum_{i=1}^{N} \frac{x_{i}-x}{d_{i}(\boldsymbol{x})} \sum_{k=1}^{K}\left(r_{i, k}-d_{i}(\boldsymbol{x})-\mu_{1}^{(\eta+1)}\right) \tilde{P}_{i, k, 1}^{(\eta)} \\
& +\frac{2}{\sigma_{2}^{2,(\eta+1)}} \sum_{i=1}^{N} \frac{x_{i}-x}{d_{i}(\boldsymbol{x})} \sum_{k=1}^{K}\left(r_{i, k}-d_{i}(\boldsymbol{x})-\mu_{2}^{(\eta+1)}\right) \tilde{P}_{i, k, 2}^{(\eta)} .
\end{aligned}
$$




$$
\begin{aligned}
& \operatorname{Eval}(10): \nabla_{y} f_{1}(\boldsymbol{x})= \\
& \frac{2}{\sigma_{1}^{2,(\eta+1)}} \sum_{i=1}^{N} \frac{y_{i}-y}{d_{i}(\boldsymbol{x})} \sum_{k=1}^{K}\left(r_{i, k}-d_{i}(\boldsymbol{x})-\mu_{1}^{(\eta+1)}\right) \tilde{P}_{i, k, 1}^{(\eta)} \\
& +\frac{2}{\sigma_{2}^{2,(\eta+1)}} \sum_{i=1}^{N} \frac{y_{i}-y}{d_{i}(\boldsymbol{x})} \sum_{k=1}^{K}\left(r_{i, k}-d_{i}(\boldsymbol{x})-\mu_{2}^{(\eta+1)}\right) \tilde{P}_{i, k, 2}^{(\eta)} .
\end{aligned}
$$

Some parts of (61), (62) and (63) can be calculated a priori, namely,

$$
\begin{aligned}
& \operatorname{Eval}(11): d_{i}(\boldsymbol{x}), i=1,2, \ldots, N \\
& \operatorname{Eval}(12): \frac{x_{i}-x}{d_{i}(\boldsymbol{x})}, i=1,2, \ldots, N \\
& \operatorname{Eval}(13): \frac{y_{i}-y}{d_{i}(\boldsymbol{x})}, i=1,2, \ldots, N \\
& \operatorname{Eval}(14): r_{i, k}-d_{i}(\boldsymbol{x})-\mu_{l}^{(\eta+1)}
\end{aligned}
$$

for $i=1,2, \ldots, N, k=1,2, \ldots, K, l=1,2$.

$$
\operatorname{Eval}(15): \sum_{k=1}^{K}\left(r_{i, k}-d_{i}(\boldsymbol{x})-\mu_{l}^{(\eta+1)}\right) \tilde{P}_{i, k, l}^{(\eta)}
$$

for $i=1,2, \ldots, N, l=1,2$.

It is easy to verify that $\operatorname{Eval}(11)$ requires $N\left(2 \mathcal{E}_{\text {sub }}+\right.$ $\left.2 \mathcal{E}_{\text {pow }}+1 \mathcal{E}_{\text {sqrt }}+1 \mathcal{E}_{\text {add }}\right)$ FLOPs, both $\operatorname{Eval}(12)$ and $\operatorname{Eval}(13)$ require $N\left(1 \mathcal{E}_{\text {sub }}+1 \mathcal{E}_{\text {div }}\right)$ FLOPs, $\operatorname{Eval}(14)$ requires $3 N K \mathcal{E}_{\text {sub }}$ FLOPs, and $\operatorname{Eval}(15)$ requires $2 N\left(K \mathcal{E}_{m u l}+(K-\right.$ $\left.1) \mathcal{E}_{a d d}\right)$. Hence, $\operatorname{Eval}(8)$ requires $2\left(N K \mathcal{E}_{\text {pow }}+N K \mathcal{E}_{\text {mul }}+\right.$ $\left.(N K-1) \mathcal{E}_{\text {add }}+1 \mathcal{E}_{\text {div }}\right)$ FLOPs, both $\operatorname{Eval}(9)$ and $\operatorname{Eval}(10)$ require $2\left(N \mathcal{E}_{m u l}+(N-1) \mathcal{E}_{\text {add }}+1 \mathcal{E}_{\text {div }}\right)$ FLOPs. Let us define $F L\left(f_{1}(\boldsymbol{x})\right)$ and $F L\left(\nabla_{\boldsymbol{x}} f_{1}(\boldsymbol{x})\right)$ to denote respectively the total number of FLOPs required to evaluate the cost function and its gradient at a certain point $\boldsymbol{x} . F L\left(f_{1}(\boldsymbol{x})\right)$ is the total FLOPs consumed in Eval(11) through Eval(14) and Eval(8), and the final result is

$$
\begin{gathered}
F L\left(f_{1}(\boldsymbol{x})\right)=(4 N K-N-2) \mathcal{E}_{\text {add }}+(3 N K+4 N) \mathcal{E}_{\text {sub }} \\
+(4 N K) \mathcal{E}_{\text {mul }}+(2 N+2) \mathcal{E}_{\text {div }}+(2 N K+2 N) \mathcal{E}_{\text {pow }}+N \mathcal{E}_{\text {sqrt }}
\end{gathered}
$$

$F L\left(\nabla_{\boldsymbol{x}} f_{1}(\boldsymbol{x})\right)$ is the total FLOPs consumed in Eval $(9)$ through Eval(15), and the final result is

$$
\begin{gathered}
\quad F L\left(\nabla_{\boldsymbol{x}} f_{1}(\boldsymbol{x})\right)=(2 N K+3 N-4) \mathcal{E}_{a d d}+(3 N K+4 N) \mathcal{E}_{\text {sub }} \\
+(2 N K+4 N) \mathcal{E}_{\text {mul }}+(2 N+4) \mathcal{E}_{\text {div }}+2 N \mathcal{E}_{\text {pow }}+N \mathcal{E}_{\text {sqrt }}
\end{gathered}
$$

Having the above knowledge, we then proceed with the complexity analysis of the BFGS quasi-Newton method in one iteration. First, we compute a search direction according to

$$
\operatorname{Eval}(16): \mathbf{s}_{j}=-\mathbf{H}_{j} \cdot \nabla_{\boldsymbol{x}} f_{1}\left(\boldsymbol{x}_{j}^{(\eta+1)}\right)
$$

which requires $4 \mathcal{E}_{m u l}+2 \mathcal{E}_{a d d}+F L\left(\nabla_{\boldsymbol{x}} f_{1}(\boldsymbol{x})\right)$ FLOPs. Note that $j$ is the iteration index and $\boldsymbol{x}_{0}^{(\eta+1)}=\boldsymbol{x}^{(\eta)}$ when $j=0$. The selection of a step size $\tau_{j}$ is done via cubic line search (Eval(17)) in the second step, which requires approximately $N_{i t r}^{L S}\left(F L\left(f_{1}(\boldsymbol{x})\right)+F L\left(\nabla_{\boldsymbol{x}} f_{1}(\boldsymbol{x})\right)\right)$ FLOPs where $N_{i t r}^{L S}$ is the number of trials used to determine a suitable step size. In the third step, we update the estimate by $\boldsymbol{x}_{j+1}^{(\eta+1)}=\boldsymbol{x}_{j}^{(\eta+1)}+\tau_{j} \mathbf{s}_{j}$, which $(\operatorname{Eval}(18))$ requires $2 \mathcal{E}_{m u l}+2 \mathcal{E}_{a d d}$ FLOPs. In the fourth step, the evaluation $(\operatorname{Eval}(19))$ of $\gamma_{j}=\nabla_{\boldsymbol{x}} f_{1}\left(\boldsymbol{x}_{j+1}^{(\eta+1)}\right)-$ $\nabla_{\boldsymbol{x}} f_{1}\left(\boldsymbol{x}_{j}^{(\eta+1)}\right)$ requires $F L\left(\nabla_{\boldsymbol{x}} f_{1}(\boldsymbol{x})\right)+2 \mathcal{E}_{\text {sub }}$ FLOPs. The fifth step approximates the Hessian matrix, which $(\operatorname{Eval}(20))$ requires in total $36 \mathcal{E}_{\text {mul }}+19 \mathcal{E}_{\text {add }}+10 \mathcal{E}_{\text {div }}$ FLOPs. Let us define $F L(\boldsymbol{x})$ to be the total number of FLOPs required to compute an estimate of $\boldsymbol{x}$ for one EM iteration. It is equal to $N_{i t r}^{Q N}$ times of the total FLOPs consumed in Eval(16) through $\operatorname{Eval}(20)$, and the final result is

$$
F L(\boldsymbol{x}) \approx N_{i t r}^{Q N}\left[\left(N_{i t r}^{L S}+2\right) F L\left(\nabla_{\boldsymbol{x}} f_{1}(\boldsymbol{x})\right)+N_{i t r}^{L S} F L\left(f_{1}(\boldsymbol{x})\right)\right]
$$

where $N_{i t r}^{Q N}$ is the number of iterations at the convergence of the quasi-Newton method.

Finally, let $N_{i t r}^{E M}$ be the number of iterations used to reach the convergence of the EM algorithm. The total FLOPs used to compute an ultimate EM estimate is

$$
F L_{E M} \approx N_{i t r}^{E M}\left[F L\left(\boldsymbol{\theta}_{m}\right)+F L(\boldsymbol{x})\right] .
$$

Under the following mild conditions:

1) the maximum iteration numbers of $N_{i t r}^{E M}, N_{i t r}^{Q N}$, and $N_{i t r}^{L S}$ are constants,

2) the elementary operations demand constant FLOPs,

3 ) both $N$ and $K$ are large,

we conclude that the complexity of the EM algorithm is of order $\mathcal{O}(N K)$ FLOPs.

\section{APPENDIX B \\ INITIALIZATION STRATEGY}

The initialization strategy in Algorithm 2 is built on the results given in [26, Sec.1.4.16], that is, the exact mean $\mu_{\mathrm{V}}$ and variance $\sigma_{\mathrm{V}}^{2}$ of $p_{V}(v)$ can be found, respectively, by

$$
\begin{gathered}
\mu_{\mathrm{V}}=\alpha_{1} \mu_{1}+\alpha_{2} \mu_{2} \\
\sigma_{\mathrm{V}}^{2}=\sum_{l=1}^{2} \alpha_{l} \sigma_{l}^{2}+\alpha_{l}\left(\mu_{l}-\mu_{\mathrm{V}}\right)^{2}
\end{gathered}
$$

Assuming an initial guess $\alpha_{2}^{(0)}=\varepsilon^{(0)}, \mu_{1}^{(0)}=0$ and replacing $\mu_{\mathrm{V}}, \alpha_{2}$ with $\tilde{\mu}_{\mathrm{V}}$ (an estimate of $\left.\mu_{\mathrm{V}}\right), \alpha_{2}^{(0)}$, respectively, in (74), yields

$$
\mu_{2}^{(0)}=\tilde{\mu}_{\mathrm{V}} / \alpha_{2}^{(0)}=\tilde{\mu}_{\mathrm{V}} / \varepsilon^{(0)} .
$$

Replacing $\sigma_{\mathrm{V}}^{2}, \alpha_{1}, \alpha_{2}, \mu_{1}, \mu_{2}$ with $\tilde{\sigma}_{\mathrm{V}}^{2}$ (an estimate of $\sigma_{\mathrm{V}}^{2}$ ), $\alpha_{1}^{(0)}, \alpha_{2}^{(0)}, \mu_{1}^{(0)}, \mu_{2}^{(0)}$, respectively, in (75) and assuming $\sigma_{2}^{2}=$ $L \cdot \sigma_{1}^{2}(L>1)$, yields

$$
\begin{aligned}
& \sigma_{1}^{2,(0)}=\frac{\tilde{\sigma}_{\mathrm{V}}^{2}-\varepsilon^{(0)}\left(1-\varepsilon^{(0)}\right)\left(\mu_{2}^{(0)}\right)^{2}}{1-\varepsilon^{(0)}+L \cdot \varepsilon^{(0)}}, \\
& \sigma_{2}^{2,(0)}=L \cdot \sigma_{1}^{2,(0)} .
\end{aligned}
$$

Since $\sigma_{1}^{2}$ and $\sigma_{2}^{2}$ are assumed to be strictly larger than 0 and $\mu_{2}$ is more likely to be positive, we take the absolute value of $\sigma_{1}^{2,(0)}, \sigma_{2}^{2,(0)}$, and $\mu_{2}^{(0)}$ in the next step. This initialization strategy can be straightforwardly extended for the cases where the measurement errors are Gaussian-Rayleigh or Gaussianexponential distributed. 


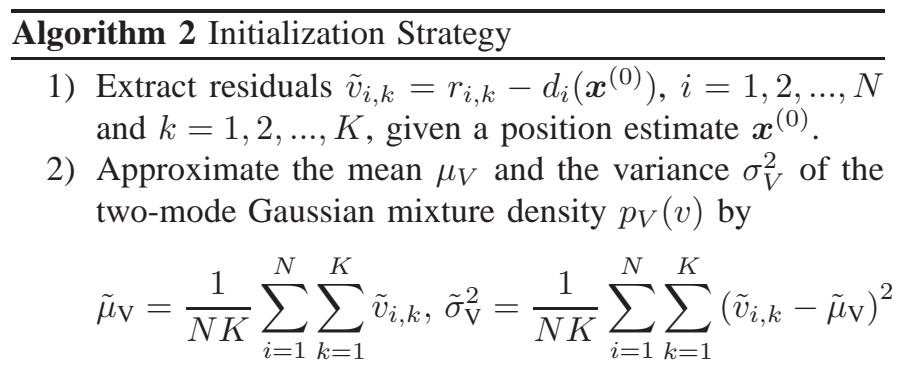

3) Let $\varepsilon^{(0)}$ vary from 0 to 1 with an increment $1 / N$ where $N$ is the number of BSs. For each $\varepsilon^{(0)}$, compute

$$
\begin{gathered}
\alpha_{1}^{(0)}=1-\varepsilon^{(0)}, \alpha_{2}^{(0)}=\varepsilon^{(0)}, \mu_{2}^{(0)}=\left|\tilde{\mu}_{\mathrm{V}} / \varepsilon^{(0)}\right|, \\
\sigma_{1}^{2,(0)}=\frac{\left|\tilde{\sigma}_{\mathrm{V}}^{2}-\varepsilon^{(0)}\left(1-\varepsilon^{(0)}\right)\left(\mu_{2}^{(0)}\right)^{2}\right|}{1-\varepsilon^{(0)}+L \cdot \varepsilon^{(0)}}, \sigma_{2}^{2,(0)}=L \cdot \sigma_{1}^{2,(0)}
\end{gathered}
$$

4) Amongst all the candidate initial guesses obtained from step 3), choose the one maximizing $\mathcal{L}_{I}\left(\boldsymbol{\theta}^{(0)} ; \mathbf{r}\right)$. To further improve the quality, we could also try different values of $L$.

\section{ACKNOWLEDGMENT}

The authors would like to thank the anonymous reviewers for their constructive and valuable comments, which improved the quality of the paper.

\section{REFERENCES}

[1] F. Gustafsson and F. Gunnarsson, "Positioning using time-difference of arrival measurements," in Proc. IEEE Int. Conf. Acoustics, Speech and Signal Processing (ICASSP), vol. 6, Hong Kong, China, Apr. 2003, pp. VI-553-556.

[2] N. Patwari, A. O. Hero III, M. Perkins, N. S. Correal, and R. J. O'Dea, "Relative location estimation in wireless sensor networks," IEEE Trans. Signal Process., vol. 51, no. 8, pp. 2137-2148, Aug. 2003.

[3] K. W. Cheung, H. C. So, W. K. Ma, and Y. T. Chan, "Least squares algorithms for time-of-arrival-based mobile location," IEEE Trans. Signal Process., vol. 52, no. 4, pp. 1121-1130, Apr. 2004.

[4] H. C. So and F. K. W. Chan, "A generalized subspace approach for mobile positioning with time-of-arrival measurements," IEEE Trans. Signal Process., vol. 55, no. 10, pp. 5103-5107, Oct. 2007.

[5] H.-W. Wei, Q. Wan, Z.-X. Chen, and S.-F. Ye, "A novel weighted multidimensional scaling analysis for time-of-arrival-based mobile location," IEEE Trans. Signal Process., vol. 56, no. 7, pp. 3018-3022, Jul. 2008.

[6] H. C. So and L. Lin, "Linear least squares approach for accurate received signal strength based source localization," IEEE Trans. Signal Process., vol. 59, no. 8, pp. 4035-4040, Aug. 2011.

[7] K. C. Ho, "Bias reduction for an explicit solution of source localization using TDOA," IEEE Trans. Signal Process., vol. 60, no. 5, pp. 21012114, May 2012.

[8] H. Wymeersch, J. Lien, and M. Z. Win, "Cooperative localization in wireless networks," IEEE Proceeding, vol. 97, no. 2, pp. 427-450, Feb. 2009.

[9] Y. Liu, Y.-H. Hu, and Q. Pan, "Distributed, robust acoustic source localization in a wireless sensor network," IEEE Trans. Signal Process., vol. 60 , no. 8, pp. 4350-4359, Aug. 2012.

[10] C. Fritsche and A. Klein, "On the performance of mobile terminal tracking in urban GSM networks using particle filters," in Proc. Eur. Signal Process. Conf. (EUSIPCO), Glasgow, Scotland, Aug. 2009, pp. 1953-1957.

[11] S. Kay, Fundamentals of Statistical Signal Processing: Estimation Theory. Englewood Cliffs, NJ: Prentice-Hall, Inc., 1993.

[12] A. M. Zoubir and R. F. Brcich, "Multiuser detection in heavy tailed noise," Digital Signal Process., vol. 12, no. 2-3, pp. 262-273, 2002.
[13] F. Yin, C. Fritsche, F. Gustafsson, and A. M. Zoubir, "TOA based robust wireless geolocation and Cramer-Rao lower bound analysis in harsh LOS/NLOS environments," IEEE Trans. Signal Process., vol. 61, no. 9, pp. 2243-2255, May 2013.

[14] A. P. Dempster, N. Laird, and D. B. Rubin, "Maximum likelihood from incomplete data via the EM algorithm," J. Roy. Statist. Soc. B, vol. 39, pp. 1-38, 1977.

[15] A. Yeredor, "The joint MAP-ML criterion and its relation to ML and to extended least-squares," IEEE Trans. Signal Process., vol. 48, no. 12, pp. 3484-3492, Dec. 2000.

[16] R. J. Kozick and B. M. Sadler, "Maximum-likelihood array processing in non-Gaussian noise with Gaussian mixtures," IEEE Trans. Signal Process., vol. 48, no. 12, pp. 3520-3535, Dec. 2000.

[17] P.-J. Chung and J. F. Bohme, "Comparative convergence analysis of EM and SAGE algorithms in DOA estimation," IEEE Trans. Signal Process., vol. 49, no. 12, pp. 2940-2949, Dec. 2001.

[18] R. D. Nowak, "Distributed EM algorithms for density estimation and clustering in sensor networks," IEEE Trans. Signal Process., vol. 51, no. 8, pp. 2245-2253, Aug. 2003.

[19] E. Ozkan, C. Fritsche, and F. Gustafsson, "Online EM algorithm for joint state and mixture measurement noise estimation," in Proc. IEEE Int. Conf. Information Fusion, Singapore, Jul. 2012, pp. 1935-1940.

[20] J. Caffery and G. L. Stuber, "Overview of radiolocation in CDMA cellular systems," IEEE Commun. Mag., vol. 36, no. 4, pp. 38-45, Apr. 1998.

[21] J. Caffery, Wireless Location in CDMA Cellular Radio Systems. Norwell, Massachusetts, USA: Kluwer Academic Publisher, 2002.

[22] H. Wymeersch, S. Marano, W. M. Gifford, and M. Z. Win, "A machine learning approach to ranging error mitigation for UWB localization," IEEE Trans. Commun., vol. 60, no. 6, pp. 1719-1728, 2012

[23] J. Riba and A. Urruela, "A non-line-of-sight mitigation technique based on ML-detection," in Proc. IEEE Int. Conf. Acoustics, Speech, and Signal Processing (ICASSP), vol. 2, Montreal, Quebec, Canada, May 2004, pp. 153-156.

[24] J. A. Bilmes, "A gentle tutorial on the EM algorithm and its application to parameter estimation for Gaussian mixture and hidden Markov models," University of California, Berkeley, Berkeley, CA, USA, Tech. Rep., 1998.

[25] J. Nocedal and S. J. Wright, Numerical optimization. New York, NY: Springer-Verlag New York, Inc., 1999.

[26] Y. Bar-Shalom, X. R. Li, and T. Kirubarajan, Estimation with Applications to Tracking and Navigation. New York, NY: John Wiley \& Sons, Inc., 2001.

[27] M. R. Gupta and Y.-H. Chen, "Theory and use of the EM algorithm," Foundations and Trends in Signal Process., vol. 4, no. 3, pp. 223-296, 2010.

[28] S. Boyd and L. Vandenberghe, Convex Optimization. New York, NY, USA: Cambridge University Press, 2004.

[29] F. Gustafsson, Statistical Sensor Fusion. Lund, Sweden: Studentlitteratur, 2012

[30] C. P. Robert and G. Casella, Monte Carlo Statistical Methods. New York, NY: Springer-Verlag, 1999.

[31] Y. T. Chan, W. Y. Tsui, H. C. So, and P. C. Ching, "Time-of-arrival based localization under NLOS conditions," IEEE Trans. Veh. Technol., vol. 55, no. 1, pp. 17-24, Jan. 2006.

[32] Y. H. Qi, H. Kobayashi, and H. Suda, "Analysis of wireless geolocation in a non-line-of-sight environment," IEEE Trans. Wireless Commun., vol. 5, no. 3, pp. 672-681, Mar. 2006.

[33] R. Zekavat and R. M. Buehrer, Handbook of Position Location. Hoboken, NJ: John Wiley \& Sons, Inc., 2011.

[34] T. Minka, "The lightspeed matlab toolbox." [Online]. Available: http://research.microsoft.com/enus/um/people/minka/software/lightspeed/ 


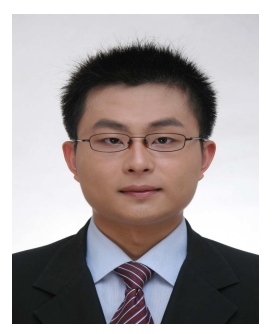

Feng Yin received the B.Sc. degree from Shanghai Jiao Tong University, China in 2008, and the M.Sc. degree from Technische Universität Darmstadt (TUD), Darmstadt, Germany in 2011.

$\mathrm{He}$ is currently working towards the Ph.D. degree in the Signal Processing Group (SPG) at Technische Universität Darmstadt. His research interests include optimal waveform design, robust estimation theory with applications to wireless geolocation, tracking and navigation.

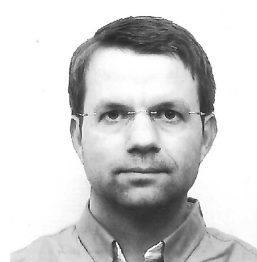

Carsten Fritsche received the Dipl.-Ing. degree in 2005 and the Dr.-Ing. degree in 2011 both in electrical engineering and information technology from Technische Universität Darmstadt, Germany. In 2011 he joined the Division of Automatic Control at Linköping University, Sweden, as a postdoctoral research fellow. Since spring 2012, he has been with IFEN GmbH, Poing, Germany, working as a systems engineer on satellite navigation related projects. His main research interest lies in the area of statistical signal processing, machine learning and sensor fusion with applications to wireless positioning and satellite navigation.

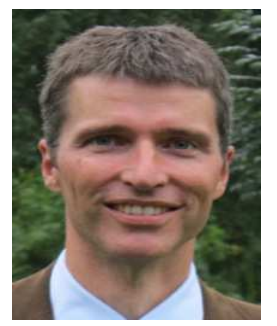

Fredrik Gustafsson is professor in Sensor Informatics at Department of Electrical Engineering, Linköping University, since 2005. He received the M.Sc. degree in electrical engineering 1988 and the Ph.D. degree in Automatic Control, 1992, both from Linköping University. During 1992-1999 he held various positions in automatic control, and 19992005 he had a professorship in Communication Systems. His research interests are in stochastic signal processing, adaptive filtering and change detection, with applications to communication, vehicular, airborne, and audio systems. He is a co-founder of the companies NIRA Dynamics (automotive safety systems), Softube (audio effects) and SenionLab (indoor positioning systems).

He was an associate editor for IEEE Transactions on Signal Processing 2000-2006 and is currently associate editor for IEEE Transactions on Aerospace and Electronic Systems and EURASIP Journal on Applied Signal Processing. He was awarded the Arnberg prize by the Royal Swedish Academy of Science (KVA) 2004, elected member of the Royal Academy of Engineering Sciences (IVA) 2007, elevated to IEEE Fellow 2011 and awarded the Harry Rowe Mimno Award 2011 for the tutorial "Particle Filter Theory and Practice with Positioning Applications", which was published in the AESS Magazine in July 2010.

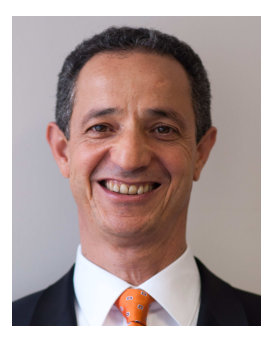

Abdelhak M. Zoubir is a Fellow of the IEEE and IEEE Distinguished Lecturer (Class 2010-2011). He received his Dr--Ing. from Ruhr- Universität Bochum, Germany in 1992. He was with Queensland University of Technology, Australia from 1992-1998 where he was Associate Professor. In 1999, he joined Curtin University of Technology, Australia as a Professor of Telecommunications and was Interim Head of the School of Electrical \& Computer Engineering from 2001 until 2003. In 2003, he moved to Technische Universität Darmstadt, Germany as Professor of Signal Processing and Head of the Signal Processing Group. His research interest lies in statistical methods for signal processing with emphasis on bootstrap techniques, robust detection and estimation and array processing applied to telecommunications, radar, sonar, automotive monitoring and safety, and biomedicine. He published over 300 journal and conference papers on these areas. Professor Zoubir was the Technical Chair of the 11th IEEE Workshop on Statistical Signal Processing (SSP 2001), General Co-Chair of the 3rd IEEE International Symposium on Signal Processing \& Information Technology (ISSPIT 2003) and of the 5th IEEE Workshop on Sensor Array and Multi-channel Signal Processing (SAM 2008). He is the General CoChair of the 14th International Workshop on Signal Processing Advances in Wireless Communications (SPAWC 2013) to be held in Darmstadt, Germany, the General Co-Chair of the 21st European Signal Processing Conference (EUSIPCO 2013) to be held in Marrakech, Morocco, and the Technical CoChair of ICASSP-14 to be held in Florence, Italy. Dr. Zoubir was an Associate Editor of the IEEE Transactions on Signal Processing (1999-2005), a Member of the Senior Editorial Board of the IEEE Journal on Selected Topics in Signal Processing (2009-2011) and he currently serves on the Editorial Boards of the EURASIP journals Signal Processing and the Journal on Advances in Signal Processing (JASP). He currently serves a three-year term as Editor-In-Chief of the IEEE Signal Processing Magazine (2012-2014). Dr. Zoubir was Past Chair (2012), Chair (2010-2011), Vice-Chair (2008-2009) and Member (20022007) of the IEEE SPS Technical Committee Signal Processing Theory and Methods (SPTM). He was a Member of the IEEE SPS Technical Committee Sensor Array and Multi-channel Signal Processing (SAM) from 2007 until 2012. He also serves on the Board of Directors of the European Association of Signal Processing (EURASIP). 